\title{
ON MEASURES INVARIANT UNDER TORI ON QUOTIENTS OF SEMI-SIMPLE GROUPS
}

\author{
MANFRED EINSIEDLER AND ELON LINDENSTRAUSS
}

\begin{abstract}
We classify invariant and ergodic probability measures on arithmetic homogeneous quotients of semisimple $S$-algebraic groups invariant under a maximal split torus in at least one simple local factor, and show that the algebraic support of such a measure splits into the product of four homogeneous spaces: a torus, a homogeneous space on which the measure is (up to finite index) the Haar measure, a product of homogeneous spaces on each of which the action degenerates to a rank one action, and a homogeneous space in which every element of the action acts with zero entropy.
\end{abstract}

\section{INTRODUCTION}

1.1. Background. It is well-known that orbits of one parameter diagonal groups such as the group $\left\{\left(\begin{array}{cc}s & \\ & s^{-1}\end{array}\right): s \in \mathbb{R}^{\times}\right\}$on the quotient space $\mathrm{SL}(2, \mathbb{Z}) \backslash \mathrm{SL}(2, \mathbb{R})$ can have very irregular closures (this remains true even if one assumes the orbits are bounded), as well as the closely interlinked fact that this action has a profusion of invariant probability measures. This phenomenon occurs more generally for actions of rank one $\mathbb{R}$-split tori (embeddings of the multiplicative group of $\mathbb{R}$ in an $\mathbb{R}$-algebraic group $\mathbf{G}$ ) on quotient spaces $\Gamma \backslash \mathbf{G}(\mathbb{R})$ (see e.g. [18]) as well as for $\mathbb{Q}_{p}$-groups.

This situation changes dramatically for the action of higher rank tori, and implicitly this has been observed already in the 1950's by Cassels and Swinnerton-Dyer [1] (though in a different, dual, language). In particular, [1, Hypothesis A] is equivalent to the existence of a bounded but non-periodic orbit of the rank 2 diagonal group $A<\mathrm{SL}(3, \mathbb{R})$ on $\mathrm{SL}(3, \mathbb{Z}) \backslash \mathrm{SL}(3, \mathbb{R})$, and Cassels and Swinnerton-Dyer state that they "tend rather to believe" that this Hypothesis is false (an interesting account of this insightful paper, as well as its connection to Margulis'

Date: August 2011.

M. E. acknowledges the support by the SNF (Grant 200021-127145). E. L. acknowledges the support of the ERC (AdG Grant 267259), the ISF (983/09) and the NSF (DMS-0800345). 
proof of the Oppenheim Conjecture, has been given by Margulis [23]). This phenomenon was independently discovered and investigated by Furstenberg, who studied subsets of $\mathbb{R} / \mathbb{Z}$ invariant under non-virtually cyclic multiplicative semigroups of integers [12]. Furstenberg also posed (though not in print) an influential conjecture regarding the possibile invariant measures for such actions, a conjecture that is still open.

One of the highlights of the theory of flows on homogeneous spaces is the work of Ratner on the action of groups $H$ generated by Adunipotent one parameter subgroups (e.g. [30, 31, 32]), which was later extended by Ratner [33] and by Margulis and Tomanov [24] to the $S$ algebraic setting we consider in this paper. This important work has had numerous applications in number theory, geometry, and other areas in mathematics. The cornerstone of Ratner's approach to the study of the action of such groups $H$ on quotient spaces $\Gamma \backslash G$ is the study of $H$-invariant and ergodic probability measures on such spaces, and in a series of papers culminating in [31] she gives a complete classification of these measures. Using this classification, Ratner has been able to resolve in full Raghunathan's Conjecture on orbit closures for the action of such groups, as well as establish the equidistribution of individual orbits for one parameter unipotent groups [32]. Special cases of Raghunathan's Conjecture were established earlier by purely topological methods by Dani and Margulis, such as in the paper [2].

With regards to the action of higher rank tori, and in particular the conjecture of Cassels and Swinnerton-Dyer quoted above, the most significant progress to date has also been achieved via the study of invariant measures, though to date a full classification of invariant measures remains elusive.

The first substantial results regarding measures invariant under higher rank abelian actions were in the context of actions on $\mathbb{R} / \mathbb{Z}$. Rudolph [35] (following some results of Lyons [21]) showed that Lebesgue measure is the only probability measure on $\mathbb{R} / \mathbb{Z}$ invariant and ergodic under the action of the multiplicative semigroup of integers generated by two relatively prime integers which has has positive ergodic theoretic entropy with respect to one of the generators of the semigroup (his result was subsequently generalized to any non-virtually cyclic semigroup by Johnson [14]) . This result is substantial in part because the condition of having entropy larger than some given positive lower bound is stable under weak* limits, which allows one to deduce interesting corollaries from this partial measure classification results; perhaps the first instance where the strategy was utilized was in Johnson and Rudolph's paper [15]. By now there are several genuinely different 
proofs of Rudolph's theorem, all require in a crucial way the entropy assumption.

Katok and Spatzier [17] were the first to give a partial measure classification result for the action of higher rank groups on homogeneous spaces, using an argument that is related to Rudolph's. However, in the context of homogeneous spaces these techniques seem to give less than they give on $\mathbb{R} / \mathbb{Z}$, and so in addition to an entropy assumption Katok and Spatzier needed to assume some mixing properties for the flow, an assumption which does not behave nicely under weak* limits.

The purpose of this paper is to give a meaningful, and usable, classification of measures invariant under higher-rank tori. This extends our earlier work with A. Katok [5] on measures invariant under the diagonal group in $\Gamma \backslash G$ for $G=\operatorname{SL}(n, \mathbb{R})$ and $\Gamma=\operatorname{SL}(n, \mathbb{Z})$, as well as the work of the second author [20] which treated the case of $G=\operatorname{SL}(2, \mathbb{Z}) \times H$. Both of these results have had applications beyond the theory of flows on homogeneous spaces: we mention in particular of the proof of Arithmetic Quantum Unique Ergodicity in [20], an estimate on the dimension of the set of exception to Littlewood's Conjecture in [5], as well as extensions of results of Linnik and Duke to number fields of higher degree by Michel, Venkatesh and the authors [10, 11].

The classification we provide is less complete than that given by Ratner because of two main reasons, one of which is inherent to the action of higher rank tori, the other due to our inability to say anything meaningful about zero entropy measures:

- Rank one subactions As mentioned above, there can be no meaningful measure classification for the action of rank one tori. Even if we are considering the action of higher rank tori on a quotient of a simple algebraic group we can encounter a situation in which the action essentially degenerates into a rank one action if the measure is not fully supported, as was pointed out first by M. Rees [34] [3, Sect. 9] who constructed irregular orbit closures for the action of the full diagonal group on a compact quotient of $\mathrm{SL}(3, \mathbb{R})$.

- Zero entropy subactions Similarly, even if there is some element of the acting group which acts with positive entropy the measure may be supported on a product of homogeneous spaces on one of which there may be an exotic zero entropy measure.

1.2. Statement of main results. Before stating our main theorem we need to set up some notations. Let $\mathbb{G}$ be a semi-simple linear algebraic group defined over $\mathbb{Q}$. Let $S$ be a finite set of places including $\infty$ if $\mathbb{G}(\mathbb{R})$ is non-compact. We define $G=\mathbb{G}\left(\mathbb{Q}_{S}\right)=\prod_{\sigma \in S} \mathbb{G}\left(\mathbb{Q}_{\sigma}\right)$, where 
$\mathbb{G}\left(\mathbb{Q}_{\sigma}\right)$ is the group of $\mathbb{Q}_{\sigma}$-points of $\mathbb{G}$ for $\sigma \in S$ and $\mathbb{Q}_{\infty}=\mathbb{R}$. Furthermore, let $\Gamma<\mathbb{G}(\mathbb{Q})$ be a lattice in $G$ commensurable with $\mathbb{G}\left(\mathcal{O}_{S}\right)$ with $\mathcal{O}_{S}$ denoting the ring $\mathbb{Z}\left[\frac{1}{p}: p \in S\right]$. We define the homogeneous space $X$ to be the quotient $X=\Gamma \backslash G$.

For every $\sigma \in S$ we suppose $\mathbb{G}$ considered over $\mathbb{Q}_{\sigma}$ has the almost direct $\mathbb{Q}_{\sigma}$-almost simple factors $\mathbf{G}_{\sigma, f}$ for $f \in F_{\sigma}$ (we use $\mathbb{G}, \mathbb{L}, \ldots$ to denote algebraic groups defined over the global field $\mathbb{Q}$ and $\mathbf{G}, \mathbf{L}, \ldots$ to denote algebraic groups defined over a local field). Take $F=\bigsqcup_{\sigma \in S} F_{\sigma}$ to be the disjoint union of the index sets and we simply write $\mathbf{G}_{f}$ for the almost direct factor of $\mathbb{G}$ over $\mathbb{Q}_{\sigma}$ (with $\sigma=\sigma(f) \in S$ uniquely determined by the index $f)$. Moreover, we write $G_{f}=\mathbf{G}_{f}\left(\mathbb{Q}_{\sigma}\right)$ for the group of $\mathbb{Q}_{\sigma}$-points of $\mathbf{G}_{f}$ for any $f \in F$.

Now let $F^{\prime} \subset F$ be a non-empty subset of the set of factors. Let $A_{f} \subset \mathbf{G}_{f}\left(\mathbb{Q}_{\sigma}\right)$, for $f \in F^{\prime}$, be a subgroup of finite index of the group of $\mathbb{Q}_{\sigma}$-points of a maximal $\mathbb{Q}_{\sigma}$-split torus $\mathbf{A}_{f}<\mathbf{G}_{f}$ defined over $\mathbb{Q}_{\sigma}$. We define $A=\prod_{f \in F^{\prime}} A_{f}$ and define the rank of $A$ as

$$
\operatorname{rank}(A)=\sum_{f \in F^{\prime}} \operatorname{dim}_{\mathbb{Q}_{\sigma(f)}}\left(A_{f}\right) .
$$

We consider $A$ as a subgroup of $G$ - in particular, $A$ acts by right translation on $X$.

Theorem 1.1. Let $\mathbb{G}$ be a semi-simple linear algebraic group over $\mathbb{Q}$, and let $S, \Gamma, X, F, F^{\prime} \subset F$, and $A$ be as above. Let $\mu$ be an $A$-invariant and ergodic probability measure on $X$ and let $p \in G$ be such that $\Gamma p \in \operatorname{supp} \mu$. Then there exists a reductive linear algebraic subgroup $\mathbb{L}$ defined over $\mathbb{Q}$ so that the following holds:

(S) (Support) The measure $\mu$ is supported on the periodic orbit $\Gamma \mathbb{L}\left(\mathbb{Q}_{S}\right) p$, and there is no smaller reductive $\mathbb{Q}$-group with this property.

(D) (Decomposition) As an algebraic group, the group $\mathbb{L}$ is the almost direct product of a $\mathbb{Q}$-anisotropic $\mathbb{Q}$-torus $\mathbb{L}_{T}$ and semi-simple algebraic $\mathbb{Q}$-subgroups $\mathbb{L}_{I}, \mathbb{L}_{R}, \mathbb{L}_{Z}$ (where some of the subgroups may be trivial). Furthermore, if we set for $t \in\{T, I, R, Z\}$ the group $A_{t}$ to be $A \cap p^{-1} \mathbb{L}_{t} p$ then

$$
\breve{A}=A_{T} A_{I} A_{R} A_{Z}
$$

has finite index in $A$.

(I) (Invariance) There exists a finite index subgroup $L_{I}<p^{-1} \mathbb{L}_{I}\left(\mathbb{Q}_{S}\right) p$ which is normalized by $A_{I}$ such that $\mu$ is $L_{I}$-invariant and for $\mu$-a.e. $x$ the orbit $x L_{I}$ is periodic.

(R) (Rank one factor) The algebraic subgroup $\mathbb{L}_{R}$ is an almost direct product $\mathbb{L}_{R}=\prod_{i} \mathbb{L}_{R, i}$ of $\mathbb{Q}$-almost simple algebraic groups, $A_{R}$ 
contains the product of the subgroups $A_{R, i}=A \cap p^{-1} \mathbb{L}_{R, i}\left(\mathbb{Q}_{S}\right) p$ as a finite-index subgroup and $\operatorname{rank}\left(A_{R, i}\right)=1$ for all $i$.

(Z) (Zero entropy) $h_{\mu}(a)=0$ for all $a \in A_{Z}$.

Using the information provided by Theorem 1.1, it is possible to provide a more explicit description of the possible $A$-invariant measures $\mu$ on $\Gamma \backslash G$ :

Corollary 1.2. Under the conditions of Theorem 1.1, and with notations as in the statement of that theorem, there are for $t \in\{T, I, R, Z\}$ probability measures $\mu_{t}$ on $\left(\mathbb{L}_{t}(\mathbb{Q}) \cap \Gamma\right) \backslash \mathbb{L}_{t}\left(\mathbb{Q}_{S}\right)$, invariant and ergodic under $p A_{t} p^{-1}$, with

(T) There is a closed subgroup $T \subset \mathbb{L}_{T}\left(\mathbb{Q}_{S}\right)$ containing $p A_{T} p^{-1}$ so that $\mu_{T}$ is T-invariant, supported on a single T-orbit

(I) $\mu_{I}$ is $p L_{I} p^{-1}$-invariant, supported on a single $p L_{I} p^{-1}$-orbit

(Z) $h_{\mu_{Z}}(a)=0$ for any $a \in p A_{Z} p^{-1}$

so that $\mu$ is (up to translations) an almost direct product of $\mu_{T}, \mu_{I}, \mu_{R}, \mu_{Z}$.

More precisely, for $t \in\{T, I, R, Z\}$ let $\tilde{\mu}_{t}$ be the natural lift of $\mu_{t}$ to $\mathbb{L}_{t}\left(\mathbb{Q}_{S}\right)$. Let $\tilde{\mu}^{\prime}$ denote the push forward of the product measure $\tilde{\mu}_{T} \times$ $\tilde{\mu}_{I} \times \tilde{\mu}_{R} \times \tilde{\mu}_{Z}$ on the direct product $\mathbb{L}_{T}\left(\mathbb{Q}_{S}\right) \times \mathbb{L}_{I}\left(\mathbb{Q}_{S}\right) \times \mathbb{L}_{R}\left(\mathbb{Q}_{S}\right) \times \mathbb{L}_{Z}\left(\mathbb{Q}_{S}\right)$ to $\mathbb{L}\left(\mathbb{Q}_{S}\right) p$ via the map $\left(g_{T}, g_{I}, g_{R}, g_{Z}\right) \mapsto g_{T} g_{I} g_{R} g_{Z} p$. Then the natural lift $\tilde{\mu}$ of $\mu$ to $\mathbb{L}\left(\mathbb{Q}_{S}\right) p$ satisfies

$$
\tilde{\mu}=\frac{1}{[A: \breve{A}]} \sum_{a \in A / \breve{A}} \tilde{\mu}^{\prime} a .
$$

Note that $(\mathrm{T})$ and $(\mathrm{I})$ above are not symmetrical: we have less control over the closed subgroup $T \subset \mathbb{L}_{T}\left(\mathbb{Q}_{S}\right)$ then we have on $L_{I}$ which we know (after conjugation) is a finite index subgroup of $\mathbb{L}_{I}\left(\mathbb{Q}_{S}\right)$. Determining which subgroups $T$ may occur is linked with difficult Diophantine questions, closely connected to the conjectures discussed in [26]; see also [40, §4.4].

We shall deduce Theorem 1.1 from the following pleasantly concise special case of that theorem:

Theorem 1.3. Let $\mathbb{G}$ be a $\mathbb{Q}$-almost simple linear algebraic group, and let $S, \Gamma, X, F, F^{\prime} \subset F$, and $A$ be as above. Let $\mu$ be an $A$-invariant and ergodic probability measure on $X$. Suppose in addition that

(1) $\mu$ is not supported on any periodic orbit $\Gamma \mathbb{L}\left(\mathbb{Q}_{S}\right) p$ for any $p \in G$ and reductive $\mathbb{Q}$-subgroup $\mathbb{L} \supsetneqq \mathbb{G}$,

(2) $\operatorname{rank}(A) \geq 2$,

(3) $h_{\mu}(a)>0$ for some $a \in A$.

\footnotetext{
${ }^{1}$ I.e. left $\Gamma \cap \mathbb{L}_{t}(\mathbb{Q})$-invariant.
} 
Then there is a finite index subgroup $L_{I}<G$ so that $\mu$ is $L_{I}$-invariant and supported on a single $L_{I}$-orbit.

Remark: For some groups $\mathbb{G}$ and $\mathbb{Q}_{\sigma}$, it may occur that there are restrictions on the possible reductive subgroup $\mathbb{L}<\mathbb{G}$ with the same $\mathbb{Q}_{\sigma}$-rank as $\mathbb{G}$ and with non-isotopic center that may simplify the statements of the results above. The nicest situation is when any such $\mathbb{L}<\mathbb{G}$ will be $\mathbb{Q}$-almost simple. In such a case, if $A$ has rank $\geq 2$ and contains a maximal $\mathbb{Q}_{\sigma}$-split torus in $\mathbb{G}\left(\mathbb{Q}_{\sigma}\right)$ the statement of Theorem 1.1 and Corollary 1.2 simplifies considerably: indeed, in such a case, if $\mu$ is an $A$-invariant and ergodic measure on $\Gamma \backslash \mathbb{G}\left(\mathbb{Q}_{S}\right)$ so that $h_{\mu}(a)>0$ for some $a \in A$ then $\mathbb{L}_{T}, \mathbb{L}_{R}, \mathbb{L}_{Z}$ are all trivial, and the measure $\mu$ is $L$ invariant and supported on a single periodic orbit of $L$. This happens in particular for $\mathbb{G}=\operatorname{SL}(n)$ for all $\mathbb{Q}_{\sigma}$, or more generally for $\mathbb{G}=\operatorname{SL}(k, \mathbb{D})$ where $\mathbb{D}$ is a division algebra of degree $\ell$ over $\mathbb{Q}$ for all $\mathbb{Q} \sigma$ for which $\mathbb{D}$ is unramified (i.e. $\mathbb{D} \otimes \mathbb{Q}_{\sigma} \cong M_{\ell \times \ell}\left(\mathbb{Q}_{\sigma}\right)$ ). These are precisely the cases considered in [5, 10]; cf. also [38].

\subsection{Some remarks about the proofs, and relation to prior} works. A key construction that was first used in the context of measure classification for diagonalizable actions by Katok and Spatzier [17] (though implicitly can be found also in the proof of Rudolph's theorem [35] on invariant measures on $\mathbb{R} / \mathbb{Z}$ ) is the construction of leafwise measures for a measure $\mu$ on $X$ : a system of measures that can be defined for orbits of arbitrary subgroups of $G$ [20], but which is particularly informative for the orbits of $A$-normalized unipotent groups $U$ which are contracted by some $a \in A$. Whether these systems of measures degenerate and become trivial is closely connected to the positivity of entropy of $\mu$, and this is precisely why the condition of positive entropy is so useful.

In broad outline, our argument is similar to that of our joint paper with Katok [5]. Using the leafwise measures, one is able to isolate the contribution of each non-divisible root $\alpha$ of $G$ to the entropy using the leafwise measures for the group $U^{\alpha}$ corresponding to the roots $\alpha$ and possibly $2 \alpha$ (if $2 \alpha$ is also a root). If there are non-commuting roots $\alpha, \beta$ which contribute nontrivially to the entropy (the "high entropy" case) then the work of Katok and the first author [3, 4] gives that $\mu$ is invariant under some one parameter unipotent group (in which case one can then apply Ratner's Measure Classification Theorem or its $S$-algebraic extensions).

If, however, there is a root $\alpha$ which contributes nontrivially to the entropy but the high entropy assumption is not satisfied (the "low 
entropy" case) a completely different argument is used which uses non-measure preserving (even non measure-class preserving!) dynamics along $U^{\alpha}$, using ideas developed in earlier works of Ratner on rigidity of horocycle flows $[27,28,29]$ (where of course the unipotent flow was measure preserving). The measure preserving action of the group $A$ merely exists in the background and ensures suitable regularity. This argument was first applied in the paper [20] by the second author, and was combined with the high entropy argument in our joint paper with Katok [5].

The main difference of the general maximally split case we consider here from the case of the diagonal group acting on $\Gamma \backslash \operatorname{SL}(n, \mathbb{R})$ analyzed in [5] is that in the $\operatorname{SL}(n, \mathbb{R})$ case, all the groups $U^{\alpha}$ were one dimensional, whereas in our case these groups $U^{\alpha}$ are multidimensional and even (if we have double roots) noncommutative. The above mentioned paper [4] provides us with a suitable generalization of the high entropy argument. Generalizing the low entropy argument proved to be quite tricky, and was carried out, specifically for this purpose, in our paper [8], which should be considered as a technical first part of the present paper.

The tools we use give some information also for more general higher rank abelian actions on homogeneous spaces. In particular, in [4] (using only the high entropy argument) it is shown that in many cases any measure invariant and ergodic under a higher rank abelian action with entropy sufficiently close to that of uniform measure must coincide with the uniform measure on this homogeneous space. However, we believe we have pushed these tools to the limit if one wants to get relatively sharp measure classification results such as Theorem 1.1 and Corollary 1.2. Beyond the class of actions we consider here new ideas are needed.

The results of this paper have been announced (in slightly different, less arithmetic form) in [7].

\section{SOME REDUCTIONS}

The purpose of this section is to show how Theorem 1.1 can be reduced to its special case Theorem 1.3. We achieve this gradually in several steps.

2.0. Reduction to the case $\Gamma e$ in the support of $\mu$. Mostly for notational convenience, by replacing $\mu$ with $\mu p^{-1}$ (i.e., the push-forward of $\mu$ under the map $x \mapsto x p^{-1}$ ) and $A$ with $p A p^{-1}$ for $p \in G$ with $\Gamma p \in \operatorname{supp} \mu$ we may assume that $\Gamma e \in \operatorname{supp} \mu$. 
2.1. Passage to finite index subgroups. We continue our reduction by showing how Theorem 1.1 for some group $A=\prod_{f \in F^{\prime}} A_{f}$ can be deduced from knowing that this theorem holds for a finite index subgroup $A^{\prime}=\prod_{f \in F^{\prime}} A_{f}^{\prime}<A$.

Suppose $\mu$ is a measure on $\Gamma \backslash G$ invariant and ergodic under $A$. Then we may write $\mu$ as

$$
\mu=\frac{1}{\left[A: A^{\prime}\right]} \sum_{a \in A / A^{\prime}} \mu^{\prime} a
$$

with $\mu^{\prime}$ invariant and ergodic under $A^{\prime}$.

Applying Theorem 1.1 on $\mu^{\prime}$ we obtain $\mathbb{Q}$-groups $\mathbb{L}, \mathbb{L}_{T}, \mathbb{L}_{I}, \mathbb{L}_{R}, \mathbb{L}_{Z}$ and finite index subgroup $L_{I}<p^{-1} \mathbb{L}_{I}\left(\mathbb{Q}_{S}\right) p$ as in that theorem.

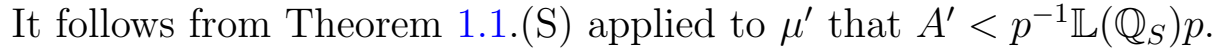
Recall that for any $f \in F^{\prime}$ both $A_{f}$ and $A_{f}^{\prime}$ are finite index subgroups of the $\mathbb{Q}_{\sigma(f)}$-points of a maximally $\mathbb{Q}_{\sigma(f)}$-split torus $\mathbf{A}_{f}$ of $\mathbf{G}_{f}$. As $A_{f}^{\prime}$ is Zariski dense in $\mathbf{A}_{f}$ we may conclude that $A_{f}$ (hence $A$ ) are contained in $p^{-1} \mathbb{L}\left(\mathbb{Q}_{S}\right) p$. Equation (2.1) implies that $\mu$ also satisfies Theorem 1.1.(S) for the same $\mathbb{L}$.

Theorem 1.1.(D) and Theorem 1.1.(R) are purely algebraic statements that do not involve $\mu^{\prime}$ and moreover the validity of these statements for $A^{\prime}$ implies elementarily that they are valid for $A$ as well. Using the relation between $\mu$ and $\mu^{\prime}$ given in (2.1) and basic properties of the ergodic theoretic entropy is easy to see that Theorem 1.1.(Z) for $\mu^{\prime}$ and $A^{\prime}$ implies the same for $\mu$ and $A$.

Thus it only remains to verify Theorem 1.1.(I) for $\mu$ and $A$. We note that if $L_{I}$ satisfies Theorem 1.1.(I) for $\mu^{\prime}$ and $A^{\prime}$ then so does any finite index subgroup of $L_{I}$, hence by replacing $L_{I}$ with

$$
\bigcap_{a \in A / A^{\prime}} a L_{I} a^{-1}
$$

we may assume that $L_{I}$ is normalized not just by $A_{I}^{\prime}$ but by $A_{I}$, and moreover since for $\mu^{\prime}$ almost every $x$, the orbit $x L_{I}$ is periodic, it follows from (2.1) that the same holds for $\mu$-almost every $x$.

2.2. Reduction to the case of $\mathbb{G}$ a $\mathbb{Q}$-almost simple group. Suppose $\mathbb{G}$ is not $\mathbb{Q}$-almost simple. We reduce Theorem 1.1 for $\mathbb{G}$ from the $\mathbb{Q}$-almost simple case by writing $\mathbb{G}$ as an almost direct product $\prod_{i=1}^{M} \mathbb{H}_{(i)}$ of $\mathbb{Q}$-almost simple groups $H_{(i)}$ and passing to the space $\tilde{\Gamma} \backslash \widetilde{G}\left(\mathbb{Q}_{S}\right)$, where $\tilde{\mathbb{G}}$ is the direct product of the groups $\mathbb{H}_{(i)}$, and $\tilde{\Gamma}$ the product of the lattices $\Gamma \cap \mathbb{H}_{(i)}\left(\mathbb{Q}_{S}\right)$ in $\mathbb{H}_{(i)}\left(\mathbb{Q}_{S}\right)$. We also assume as we may (see $\S 2.0)$ that $\Gamma e \in \operatorname{supp} \mu$. 
Let $\psi: \tilde{\mathbb{G}} \rightarrow \mathbb{G}$ be the obvious isogeny. Setting $\tilde{G}=\tilde{\mathbb{G}}\left(\mathbb{Q}_{S}\right)$, it is clear that $\psi$ also induces finite-to-one maps $\tilde{G} \rightarrow G$ and $\tilde{\Gamma} \backslash \tilde{G} \rightarrow$ $\Gamma \backslash G$ that will also be denoted by $\psi$. To avoid confusion, we make a notational distinction between the algebraic $\mathbb{Q}$-subgroup $\mathbb{H}_{(i)}$ of $\mathbb{G}$ and the isomorphic subgroup $\tilde{\mathbb{H}}_{(i)}$ of $\tilde{\mathbb{G}}$. By construction (and after relabeling indices if necessary), the group $A$ can be written as a product group $\prod_{i=1}^{M^{\prime}} A_{(i)}$ with $A_{(i)}$ a nontrivial subgroup of $\mathbb{H}_{(i)}\left(\mathbb{Q}_{S}\right)$. Let $\tilde{A}$ be the corresponding product group in $\tilde{G}$. Note that while in general $\psi(\tilde{G})$ may have finite index in $G$, the way things have been set up assures that $\psi(\tilde{A})=A$. Since $\mu$ is $A$-ergodic and $A \subset \psi(\tilde{G})$ it follows that $\mu$ is supported on a single orbit of $\psi(\tilde{G})$. Since $\Gamma e \in \operatorname{supp} \mu$ we conclude that $\operatorname{supp} \mu \subset \Gamma \psi(\tilde{G})$.

The collection of probability measures $\nu$ on $\tilde{\Gamma} \backslash \tilde{G}$ with $\psi_{*} \nu=\mu$ is a convex compact set, which is clearly nonempty if we assume $\mu$ is supported on $\Gamma \psi(\tilde{G})$. By averaging, we can find inside this collection an $\tilde{A}$-invariant measure, and by taking ergodic component we deduce that there is an $\tilde{A}$-ergodic and invariant probability measure $\tilde{\mu}$ on $\tilde{\Gamma} \backslash \tilde{G}$ so that $\psi_{*} \tilde{\mu}=\mu$. Since $\tilde{\mu}$ is ergodic under the action of a product group of a product space, it must be of the form $\tilde{\mu}=\tilde{\mu}_{(1)} \times \cdots \times \tilde{\mu}_{(M)}$ with $\tilde{\mu}_{(i)}$ for $i \leq M^{\prime}$ an $\tilde{A}_{(i)}$-ergodic and invariant measure on $\tilde{\Gamma}_{(i)} \backslash \tilde{\mathbb{H}}_{(i)}\left(\mathbb{Q}_{S}\right)$ and $\tilde{\mu}_{(i)}$ atomic, supported on a single point, for $i>M^{\prime}$.

Assuming that Theorem 1.1 has been established for $\mathbb{Q}$-almost simple groups, we have for each $i \leq M^{\prime}$ four $\mathbb{Q}$-groups $\tilde{\mathbb{L}}_{t}^{(i)}$ of $\tilde{\mathbb{H}}_{(i)}$ for $t \in$ $\{T, I, R, Z\}$ that satisfy all the conditions of Theorem 1.1 for $\tilde{\mu}_{(i)}$ on $\tilde{\Gamma}_{(i)} \backslash \mathbb{H}_{(i)}\left(\mathbb{Q}_{S}\right)$. The $\mathbb{Q}$-groups $\mathbb{L}_{t}=\psi\left(\prod_{i=1}^{M^{\prime}} \tilde{\mathbb{L}}_{t}^{(i)}\right)$ for $t \in\{T, I, R, Z\}$ are now natural candidates for satisfying the conditions of Theorem 1.1 for $\mathbb{G}$.

Verifying that this choice satisfies (S), (D), (I), (R) of Theorem 1.1 is straightforward (for (I), and in obvious notations, $A_{I}$ may contain $\psi\left(\prod_{i} \tilde{A}_{I}^{(i)}\right)$ as a nontrivial finite index subgroup, hence may hypothetically fail to normalize $L_{I}:=\psi\left(\prod_{i} \tilde{L}_{I}^{(i)}\right)$, but replacing $L_{I}$ by a finite index subgroup if necessary as in (2.2) alleviates this minor nuisance).

To see that $(\mathrm{Z})$ holds, i.e. that for every $a \in A_{Z}$ the entropy $h_{\mu}(a)=$ 0 , we note first that it is enough to check this for $a$ in the (at worst) finite index subgroup $\psi\left(\prod_{i} A_{Z}^{(i)}\right)$, i.e. for $a=\psi\left(\left(a_{1}, \ldots, a_{M^{\prime}}, e, \ldots, e\right)\right)$ with $a_{i} \in \tilde{A}_{Z}^{(i)}$. Since $\psi: \tilde{\Gamma} \backslash \tilde{G} \mapsto \Gamma \backslash G$ is finite-to-one and $\psi_{*} \tilde{\mu}=\mu$

$$
h_{\mu}(a)=h_{\tilde{\mu}}\left(\left(a_{1}, \ldots, a_{M^{\prime}}, e, \ldots, e\right)\right)=\sum_{i=1}^{M^{\prime}} h_{\tilde{\mu}_{(i)}}\left(a_{i}\right)=0
$$


where the last equality follows from $a_{i} \in \tilde{A}_{Z}^{(i)}$ and the fact that $\tilde{\mu}_{(i)}$ satisfies Theorem 1.1.(Z).

2.3. Completing the reduction. Our strategy in reducing Theorem 1.1 from Theorem 1.3 is simple: we find in $\mathbb{G}$ a minimal reductive subgroup $\mathbb{L}$ so that $\Gamma \mathbb{L}\left(\mathbb{Q}_{S}\right) p$ supports $\mu$ for some $p \in G$. Assuming as we may that $\Gamma e \in \operatorname{supp} \mu$, it is enough to take $p=e$. We show that $\mathbb{L}$ does not have any $\mathbb{Q}$-characters, split $\mathbb{L}$ into an almost direct product of a torus $\mathbb{L}_{T}$ and a semisimple group $\mathbb{L}_{s s}$, and reduce the study of $\mu$ to study an $A \cap \mathbb{L}_{s s}\left(\mathbb{Q}_{S}\right)$-ergodic component of $\mu$ on $\left(\Gamma \cap \mathbb{L}_{s s}(\mathbb{Q})\right) \backslash \mathbb{L}_{s s}\left(\mathbb{Q}_{S}\right)$ which we show also satisfies the conditions of Theorem 1.1.

Lemma 2.1. Let $\mathbb{G}$ be a semisimple $\mathbb{Q}$-group, $A, G, S$ and $\Gamma$ as in Theorem 1.1 and $\mu$ an A-invariant and ergodic probability measure on $\Gamma \backslash G$ with $\Gamma e \in \operatorname{supp} \mu$. Let $\mathbb{L}$ be a $\mathbb{Q}$-subgroup of $\mathbb{G}$ so that $\mu\left(\Gamma \mathbb{L}\left(\mathbb{Q}_{S}\right)\right)=1$. Then there is a normal $\mathbb{Q}$-subgroup $\mathbb{L}^{\prime} \triangleleft \mathbb{L}$, without $\mathbb{Q}$-characters, for which $\operatorname{supp} \mu \subset \Gamma \mathbb{L}^{\prime}\left(\mathbb{Q}_{S}\right)$.

Note in particular that since normal subgroups of reductive groups are reductive, Lemma 2.1 implies that if $\mathbb{L}$ is the smallest reductive $\mathbb{Q}$-group so that $\operatorname{supp} \mu \subset \Gamma \mathbb{L}\left(\mathbb{Q}_{S}\right)$ then $\mathbb{L}$ does not have $\mathbb{Q}$-characters.

Proof. Suppose $\chi$ is a $\mathbb{Q}$-character of $\mathbb{L}$, and let $L=\mathbb{L}\left(\mathbb{Q}_{S}\right)$. If $\ell=$ $\left(\ell_{\sigma}\right)_{\sigma \in S} \in L$ with $\ell_{\sigma} \in \mathbb{L}\left(\mathbb{Q}_{\sigma}\right)$ define

$$
|\chi|(\ell)=\prod_{\sigma \in S}\left|\chi\left(\ell_{\sigma}\right)\right|_{\sigma} .
$$

This is a group homomorphism $L \rightarrow \mathbb{R}$, and moreover $|\chi|(\gamma)=1$ for every $\gamma \in \mathbb{L}\left(\mathcal{O}_{S}\right)$. Since $\Gamma_{L}:=\Gamma \cap \mathbb{L}(\mathbb{Q})$ is commensurable with $\mathbb{L}\left(\mathcal{O}_{S}\right)$ we see that $\Gamma_{L}$ is in the kernel of $|\chi|$, hence $|\chi|$ is defined on the points ${ }^{2}$ of $\Gamma L$. The group $A$ has to be in the kernel of $|\chi|$ since if there were an element $a_{0} \in A$ with $\chi\left(a_{0}\right)>1$ then for any $x \in \Gamma L$ we would have $|\chi|\left(x a_{0}^{n}\right) \rightarrow \infty$ contradicting Poincare recurrence. It follows that $\chi$ itself must be trivial on $A \cap \mathbb{G}\left(\mathbb{Q}_{\sigma}\right)$ for every $\sigma \in S$, hence $A$ is contained in the proper normal $\mathbb{Q}$-subgroup $\mathbb{L}^{\prime}=(\mathbb{L} \cap \operatorname{ker} \chi) \triangleleft \mathbb{L}$. By ergodicity of $\mu$, it follows that $A$ has to be supported on a single orbit $\Gamma L^{\prime}\left(\mathbb{Q}_{S}\right) p=\Gamma p \mathbb{L}^{\prime}\left(\mathbb{Q}_{S}\right)$ for some $p \in L$, in contradiction to choice of $\mathbb{L}$.

We now continue with the reduction. Suppose $\mathbb{L}$ is as in Lemma 2.1. We split the reductive $\mathbb{Q}$-group $\mathbb{L}$ as the semi-direct product of a $\mathbb{Q}$ torus $\mathbb{L}_{T}$ and a semisimple $\mathbb{Q}$-group $\mathbb{L}_{s s}$; since $\mathbb{L}$ has no $\mathbb{Q}$-characters,

\footnotetext{
${ }^{2}$ As usual, we think of $\Gamma L$ as a collection of points in $\Gamma \backslash G$; perhaps $\Gamma \backslash \Gamma L$ might have been more accurate but this (mis)usage is standard.
} 
the torus $\mathbb{L}_{T}$ is $\mathbb{Q}$-anisotropic. Since we have assumed that $\mu$ is supported in $\Gamma \mathbb{L}\left(\mathbb{Q}_{S}\right)$, it is clear that $A<\mathbb{L}\left(\mathbb{Q}_{S}\right)$, and since we are allowed to replace $A$ by a finite index subgroup by $\S 2.1$ we can assume that in fact $A<\mathbb{L}_{T}\left(\mathbb{Q}_{S}\right) \mathbb{L}_{s s}\left(\mathbb{Q}_{S}\right)$.

We aim to establish two important facts about how $A$ lies in the product $\mathbb{L}_{T}\left(\mathbb{Q}_{S}\right) \mathbb{L}_{s s}\left(\mathbb{Q}_{S}\right)$ :

(1) $A_{T}:=A \cap \mathbb{L}\left(\mathbb{Q}_{S}\right)$ and $A_{s s}:=A \cap \mathbb{L}\left(\mathbb{Q}_{s s}\right)$ satisfies that $A_{T} A_{s s}$ is a finite index subgroup of $A$.

(2) $A_{s s}$ (or more precisely, a finite index subgroup of $A_{s s}$ ) satisfies the conditions of Theorem 1.1 for $\mathbb{L}_{s s}$, i.e. if we decompose for each $\sigma \in S$ the group $\mathbb{L}_{s s}$, considered now as a $\mathbb{Q}_{\sigma}$-group, as the almost direct product of almost simple $\mathbb{Q}_{\sigma}$-groups $\mathbf{L}_{j}$ with $j \in J_{\sigma}$ and set $J=\bigsqcup_{\sigma \in S} J_{\sigma}$ we have that

$$
\prod_{j \in J}\left(A \cap \mathbf{L}_{j}\left(\mathbb{Q}_{\sigma(j)}\right)\right)
$$

is a finite index subgroup in $A_{s s}$.

It will be helpful to make a notational distinction between the $\mathbb{Q}$ group $\mathbb{L}$ and the same group considered as a $\mathbb{Q}_{\sigma}$-group which we denote by $\mathbf{L}$ (and similarly for $\mathbb{L}_{T}$ etc.); to make notations less cumbersome we keep the dependence on $\sigma$ implicit.

For any $f \in F$ let $\sigma \in S$ be the associated valuation and consider the $\mathbb{Q}_{\sigma}$-group $\mathbf{G}_{f} \cap \mathbf{L}$. Since $\mathbf{G}_{f}$ is normal in $\mathbf{G}$, this intersection is a normal subgroup of a reductive group hence reductive. Moreover, its decomposition into torus and semisimple parts is given by ${ }^{3}$

$$
\mathbf{G}_{f} \cap \mathbf{L}=\left(\mathbf{G}_{f} \cap \mathbf{L}_{T}\right) \cdot\left(\mathbf{G}_{f} \cap \mathbf{L}_{s s}\right) .
$$

Suppose now $f \in F^{\prime}$, and $\sigma=\sigma(f)$ the appropriate place. Let $\mathbf{A}_{f}$ be a maximal $\mathbb{Q}_{\sigma}$-split torus as in the setup of Theorem 1.1. By definition of $\mathbb{L}$ (and the assumptions that $e \in \operatorname{supp} \mu$ ) it follows that $\mathbf{A}_{f}<\mathbf{L} \cap \mathbf{G}_{f}$. Equation (2.3) allows us to project $\mathbf{A}_{f}$ to split $\mathbb{Q}_{\sigma}$-tori in $\mathbf{L}_{T} \cap \mathbf{G}_{f}$ and $\mathbf{L}_{s s} \cap \mathbf{G}_{f}$; since $\mathbf{A}_{f}$ is a maximal split torus it must coincide with a product of these projections, hence $\left(A_{f} \cap L_{T}\right)\left(A_{f} \cap L_{s s}\right)$ has finite index in $A_{f}$. Taken over all $f \in F^{\prime}$ this implies Claim (1) above. Similarly, for every $f \in F^{\prime}$, and taking $J_{f} \subset J_{\sigma(f)}$ to be the indices of all almost

\footnotetext{
${ }^{3}$ Indeed, decompose $\mathbf{G}_{f} \cap \mathbf{L}$ as a product of a $\mathbb{Q}_{\sigma}$-torus $\tilde{\mathbf{L}}_{T}$ and a semisimple $\mathbb{Q}_{\sigma}$-group $\tilde{\mathbf{L}}_{s s}$. The group $\mathbf{L}_{s s} \cap \mathbf{G}_{f}$ is a normal subgroup of a semisimple group, hence semisimple, hence $\mathbf{L}_{s s} \cap \mathbf{G}_{f} \subset \tilde{\mathbf{L}}_{s s}$. On the other hand, $\tilde{\mathbf{L}}_{s s}$ is generated by commutators of elements $\tilde{\mathbf{L}}_{s s} \subset \mathbf{L}$ hence is contained in $\mathbf{L}_{s s}$ and we conclude that $\mathbf{L}_{s s} \cap \mathbf{G}_{f}=\tilde{\mathbf{L}}_{s s}$. Similarly $\mathbf{L}_{T} \cap \mathbf{G}_{f}$ commutes with $\tilde{\mathbf{L}}_{s s}$ hence is contained in $\tilde{\mathbf{L}}_{T}$; on the other hand, $\tilde{\mathbf{L}}_{T}$ commutes with $\mathbf{L}_{s s} \cap \mathbf{G}_{f}$ as well as with all $\mathbf{G}_{f^{\prime}}$ for $f^{\prime} \in F_{\sigma} \backslash\{f\}$, hence $\tilde{\mathbf{L}}_{T}$ commutes with $\mathbf{L}_{s s}$, hence is contained in $\mathbf{L}_{T}$.
} 
simple $\mathbb{Q}_{\sigma(f)}$-groups appearing in the decomposition of $\mathbf{L} \cap \mathbf{G}_{f}$, it follows from the maximallity of rank of $A_{f}$ that $\prod_{j \in J_{f}}\left(A_{f} \cap \mathbf{L}_{j}\left(\mathbb{Q}_{\sigma(f)}\right)\right)$ is a finite index subgroup of $A_{f} \cap L_{s s}$, and moreover each $\mathbf{A}_{f} \cap \mathbf{L}_{j}$ is a maximal split $\mathbb{Q}_{\sigma(f)}$-torus in $\mathbf{L}_{j}$, establishing Claim (2).

In view of Claims (1) and (2) and $\S 2.1$ we may as well assume that $A=A_{T} A_{s s}$ and that $A_{s s}=\prod_{j \in J}\left(A \cap \mathbf{L}_{j}\left(\mathbb{Q}_{\sigma(j)}\right)\right)$. Assuming Theorem 1.1 were already established for the action of $A_{s s}$ on $(\Gamma \cap$ $\left.\mathbb{L}_{s s}(\mathbb{Q})\right) \backslash L_{s s}$ we are now in a position to show Theorem 1.1 applies to $\mu$. Since $\operatorname{dim}\left(\mathbb{L}_{s s}\right)<\operatorname{dim}(\mathbb{G})$ it is permissible to make this assumption (and argue inductively); alternatively, one can apply $\S 2.2$ on $\mathbb{L}_{s s}$ and apply Theorem 1.3 directly.

Let $\mu=\int_{X} \mu_{x}^{\mathcal{E}} d \mu(x)$ be the decomposition of $\mu$ into its ergodic components with respect to $A_{s s}$. For any $a_{0} \in A$ the action of $A_{s s}$ on the measure $\mu_{x}^{\mathcal{E}}$ is measure theoretically conjugate to the action on the translated measure $\mu_{x}^{\mathcal{E}} a_{0}$; by the construction of ergodic components this translated measure can also be identified with $\mu_{x a_{0}}^{\mathcal{E}}$. In particular, for any $a \in A_{s s}$, we have an equality of entropies

$$
h_{\mu_{x}^{\mathcal{E}}}(a)=h_{\mu_{x a_{0}}^{\mathcal{E}}}(a),
$$

hence by ergodicity of $\mu$ and using the fact that entropy is an afine quantity i.e. $h_{\mu}(a)=\int h_{\mu_{x}}(a) d \mu(x)$ we have that for any $a \in A_{s s}$

$$
h_{\mu_{x}^{\mathcal{\varepsilon}}}(a)=h_{\mu}(a) \quad \mu \text {-a.e.. }
$$

As a function of $a$, for any $A_{s s}$-invariant measure $\nu$ on $X$, the entropy $h_{\mu}(a)$ is continuous ${ }^{4}$, hence there is a $x_{0} \in \operatorname{supp} \mu$ so that $x_{0} A$ is dense in supp $\mu$ and for which (2.4) holds for all $a \in A_{s s}$.

Applying Theorem 1.1 on $\mu_{x_{0}}^{\mathcal{E}}$ we get groups $\tilde{\mathbb{L}}_{T}, \tilde{\mathbb{L}}_{I}, \tilde{\mathbb{L}}_{R}, \tilde{\mathbb{L}}_{Z}$ and using (2.4) it is easy to verify that $\mathbb{L}_{T} \cdot \tilde{\mathbb{L}}_{T}, \tilde{\mathbb{L}}_{I}, \tilde{\mathbb{L}}_{R}, \tilde{\mathbb{L}}_{Z}$ satisfy Theorem 1.1 for $\mu$.

2.4. Proof of Corollary 1.2. Corollary 1.2 follows from Theorem 1.1 using very similar arguments to the arguments that were used above in the reduction of Theorem 1.1 from Theorem 1.3.

To begin with, it is clear that without loss of generality we may assume that $p=e$ and $\mathbb{L}=\mathbb{G}$ in both Corollary 1.2 and Theorem 1.1. Let $\breve{A}$ be as in (D) of Theorem 1.1. Since $[A: \breve{A}]<\infty$ we may write $\mu$ as

$$
\mu=\frac{1}{[A: \breve{A}]} \sum_{a \in A / \breve{A}} \mu^{\prime} a
$$

\footnotetext{
${ }^{4}$ This is not an abstract property of entropy of individual element in a commutative group, but is a well-known consequence of the relation between entropy and dimension of leafwise measures, a connection explained in $\S 4$.
} 
with $\mu^{\prime}$ an $\breve{A}$-ergodic and invariant measure. Define for $t \in\{T, I, R, Z\}$ the discrete group $\Gamma_{t}=\mathbb{L}_{t}(\mathbb{Q}) \cap \Gamma$ and as in Section 2.2 it is possible to lift $\mu^{\prime}$ to an $\breve{A}$-invariant and ergodic measure $\mu^{\prime \prime}$ on

$$
\prod_{t \in\{T, I, R, Z\}}\left(\Gamma_{t} \backslash \mathbb{L}_{t}\left(\mathbb{Q}_{S}\right)\right)
$$

Since $\breve{A}=\prod_{t \in\{T, I, R, Z\}} A_{t}$ is a product group acting ergodically on a product space it follows that $\mu^{\prime \prime}$ is a product of $A_{t}$-invariant and ergodic measure $\mu_{t}^{\prime \prime}$ on $\Gamma_{t} \backslash \mathbb{L}_{t}\left(\mathbb{Q}_{S}\right)$ where $t \in\{T, I, R, Z\}$.

The quotient $\Gamma_{T} \backslash \mathbb{L}_{T}\left(\mathbb{Q}_{S}\right)$ is a finite dimensional compact abelian group, and an $A_{T}$ invariant and ergodic measure has to be the periodic measure on a single periodic orbit of a closed (but not necessarily algebraic) subgroup of $\mathbb{L}_{T}\left(\mathbb{Q}_{S}\right)$. Since every $a \in A_{Z}$ acts with zero entropy on $\mu$, hence on $\mu^{\prime}$, hence on $\mu^{\prime \prime}$, the measure $\mu_{Z}^{\prime \prime}$ has entropy zero with respect to every $a \in A_{Z}$. Regarding $\mu_{I}^{\prime \prime}$ we already know it is invariant under a finite index subgroup $L_{I}<\mathbb{L}_{I}\left(\mathbb{Q}_{S}\right)$; since $A_{I} \cap L_{I}$ is of finite index in $A_{I}$ we can find a finite index subgroup $L_{I}^{\prime}<L_{I}$ normalized by $A_{I}$ and then $\mu_{I}^{\prime \prime}$ will be invariant under the group $A_{I} \cdot L_{I}^{\prime}$. By $A_{I}$-ergodicity of $\mu_{I}^{\prime \prime}$, the measure $\mu_{I}^{\prime \prime}$ is supported on a single orbit of this group. This decomposition we have established for $\mu^{\prime \prime}$ is equivalent to the statement of the corollary (where in order to avoid passing to a finite index subgroup of $\Gamma \cap \mathbb{L}(\mathbb{Q})$ the structure on $\mu$ was given in terms of its lift $\tilde{\mu}$ to $\mathbb{L}\left(\mathbb{Q}_{S}\right)$ ).

2.5. Some variations. The reader may wonder why we insist on working over the minimal local fields $\mathbb{R}$ or $\mathbb{Q}_{p}$ (and not over $\mathbb{C}$ or finite field extensions of $\mathbb{Q}_{p}$ ). The following example shows that Theorem 1.1 and Corollary 1.2 as stated do not hold over larger local fields; indeed, essentially the best way to understand the action of these bigger tori is by reducing to the $\mathbb{R}$ or $\mathbb{Q}_{p}$ case.

Example 2.2. Let $X=\mathrm{SL}(k, \mathbb{Z}[i]) \backslash \mathrm{SL}(k, \mathbb{C})$, let $T$ be the group of diagonal matrices, and let $A \subset T$ be the subgroup of real diagonal matrices. Let $\nu$ be the Haar measure of $X_{\mathbb{R}}=\operatorname{SL}(k, \mathbb{Z}) \backslash \operatorname{SL}(k, \mathbb{R})$. We view $X_{\mathbb{R}}$ as a subset of $X$, so that $\nu$ is a measure on $X$. Let $M \subset T$ be the maximal compact subgroup that consists of all diagonal matrices with entries of absolute values one along the diagonal. Then $\mu=\int_{M} \nu h \mathrm{~d} m_{M}(h)$ is a $T$-invariant and ergodic measure. Note that $M \mathrm{SL}(k, \mathbb{R})$ is not a subgroup of $\mathrm{SL}(k, \mathbb{C})$.

Of course, this situation is not drastically different than that of Corollary 1.2. Instead of the finite average we had to take in that 
corollary, in Example 2.2 one takes an average over a compact group that commute with the $\mathbb{R}$-split torus $A$.

The analysis of this seemingly more general scenario is easily achieved by restriction of scalars: simply treating e.g. in this particular case the group $\operatorname{SL}(k, \mathbb{C})$ as an algebraic group over $\mathbb{R}$. Then $T$ becomes the group of $\mathbb{R}$-points of a maximal torus defined in $\operatorname{SL}(k, \mathbb{C})$ and $A$ becomes the group of $\mathbb{R}$-points of a maximal $\mathbb{R}$-split subtorus. In this formulation the above example also shows why we restrict ourselves to the maximal $\mathbb{R}$-split subtorus.

We conclude that the classification of $A$-invariant and $T$-invariant measures are essentially equivalent: Any $A$-invariant and ergodic measure gives rise to an $T$-invariant and ergodic measure just as in the example, and any $T$-invariant and ergodic measure can be decomposed into $A$-invariant and ergodic measures, and a relatively straightforward analysis shows that these ergodic components fit together into a measure of a type similar to that presented in Example 2.2.

\section{SEMI-SIMPLE LINEAR ALGEBRAIC GROUPS AND THEIR ROOTS}

3.1. Semi-simple linear algebraic groups. We recall some basic properties of algebraic groups, and refer the reader to [36]. We use this theory as a natural framework that makes no distinction between the real and $p$-adic numbers.

Let $\mathbb{Q}_{\infty}=\mathbb{R}$ and let $\sigma$ be $\infty$ or a prime $p$ so that $\mathbb{Q}_{\sigma}$ is either $\mathbb{R}$ or $\mathbb{Q}_{p}$. Let $|\cdot|_{\sigma}$ denote the absolute value if $\sigma=\infty$ or the $p$-adic norm if $\sigma=p$.

Let $G=\mathbf{G}\left(\mathbb{Q}_{\sigma}\right)$ be the $\mathbb{Q}_{\sigma}$-points of a semi-simple linear algebraic group $\mathbf{G}$ defined over $\mathbb{Q}_{\sigma}$ and let $A \subset G$ be the subgroup of $\mathbb{Q}_{\sigma}$-points of a maximal $\mathbb{Q}_{\sigma^{-}}$split torus in $\mathbf{G}$. Then $A \cong\left(\mathbb{Q}_{\sigma}^{\times}\right)^{k}$ were $k$ is the $\mathbb{Q}_{\sigma^{-}}$ rank of $\mathbf{G}$ and the group of characters $\chi: A \rightarrow \mathbb{Q}_{\sigma}^{\times}$is isomorphic to $\mathbb{Z}^{k}$. A subgroup $U<G$ is unipotent if for every $g \in U, g-e$ is a nilpotent matrix, i.e. for some $n,(g-e)^{n}=0$ where $e$ is identity. A subgroup $H$ is said to be normalized by $g \in G$ if $g_{H} g^{-1}=H$; $H$ is normalized by $L<G$ if it is normalized by every $g \in L$; and the normalizer $N_{G}(H)$ of $H$ is the group of all $g \in G$ normalizing it. Furthermore, $N_{G}^{1}(H)$ denotes the subgroup of the normalizer that in addition also preserves the Haar measure on $H$. Similarly, $g$ centralizes $H$ if $g h=h g$ for every $h \in H$, and we set $C_{G}(H)$, the centralizer of $H$ in $G$, to be the group of all $g \in G$ centralizing $H$.

In the context of the paper we will use the structure discussed in this section as well as Sections 3.2-3.3 for one of the factors $\mathbf{G}=\mathbf{G}_{f}$ of 
$\mathbb{G}$ considered over $\mathbb{Q}_{\sigma}$ with $f \in F^{\prime}$ so that the subgroup $A$ considered here will be the subgroup $A_{f}<A$ as in the introduction or Section 3.4.

Let $\mathfrak{g}$ be the Lie algebra of $G$ and let

$$
\operatorname{Ad}_{g}: \mathfrak{g} \rightarrow \mathfrak{g} \text { for } g \in G
$$

be the adjoint representation of $G$ on $\mathfrak{g}$. Let $\Phi$ denote the set of restricted roots, i.e. all non-trivial characters $\alpha: A \rightarrow \mathbb{Q}_{\sigma}^{\times}$such that there exists a nontrivial (restricted) root space $\mathfrak{u}^{\alpha} \subset \mathfrak{g}$ with

$$
\operatorname{Ad}_{a}(u)=\alpha(a) u \text { for } u \in \mathfrak{u}^{\alpha} \text { and } a \in A \text {. }
$$

We write $\mathfrak{g}^{0}$ for the Lie algebra of the centralizer of $A$, so that

$$
\mathfrak{g}=\mathfrak{g}^{0} \oplus \bigoplus_{\alpha \in \Phi} \mathfrak{u}^{\alpha}
$$

Since $\mathbb{Q}_{\sigma}$ is not algebraically closed, it may happen, as for instance for the algebraic group $\mathrm{SU}(n, 1)$ over $\mathbb{R}$ with $n \geq 2$, that $\alpha, 2 \alpha \in \Phi$ are roots, see also $[36,15.3 .9]$. We say a root $\alpha$ is indivisible if $\frac{1}{2} \alpha$ is not a root. For any indivisible root $\alpha$ there exists an algebraic unipotent subgroup $U^{\alpha}$ whose Lie algebra is the sum of the root spaces $\mathfrak{u}^{\alpha} \oplus \mathfrak{u}^{2 \alpha}$ (where $\mathfrak{u}^{2 \alpha}=\{0\}$ is allowed), see [36, 15.4].

By embedding the group of characters into a real vector space $V$ of dimension $k$ as a lattice, we can identify the roots with elements of $V$. Furthermore, we equip $V$ with an inner product $(\cdot, \cdot)$ such that the natural action of the Weyl group $W=W(G, A)$ of $G$ and $A$ leaves $(\cdot, \cdot)$ invariant. We need a few more fundamental properties of the Weyl group $W$. The Weyl group $W$ acts naturally on $A$, which induces an action on the characters of $A$ (the action on $V$ is the linear extension of this action). The set $\Phi$ of roots is invariant. For every root $\alpha$ there is an associated element $s_{\alpha} \in W$, its action on $V$ is an orthogonal reflection defined by

$$
s_{\alpha}(v)=v-2(\alpha, \alpha)^{-1}(\alpha, v) \alpha \text { for } v \in V .
$$

For $\alpha \neq \beta \in \Phi$ with $(\alpha, \beta)>0$ the number $m=2(\alpha, \alpha)^{-1}(\alpha, \beta)$ is an integer and $\beta, \beta-\alpha, \ldots, \beta-m \alpha=s_{\alpha}(\beta) \in \Phi$ are all roots. As is known (and we will recall below), every root comes naturally with a subtorus of $A$ of which we will use one element as described in the next lemma, which will be proved in $§ 3.3$.

Lemma 3.1. For every root $\alpha \in \Phi$ there exists an element $a_{\alpha} \in A$ with $\log \left|\beta\left(a_{\alpha}\right)\right|_{\sigma}=c_{\alpha}(\alpha, \beta)$ (for some constant $c_{\alpha}>0$ ). In fact, one can find a homomorphism $\phi$ from $\mathrm{SL}_{2}$ into $\mathbf{G}$ so that a given element of $\mathfrak{u}^{\alpha}$ is the image of an upper unipotent element of $\mathfrak{s l}_{2}$ and so that $a_{\alpha} \in A$ 
is the image of a diagonal element in $\mathrm{SL}_{2}$ with the first eigenvalue $t$ satisfying $|t|_{\sigma}>1$.

3.2. The Lie algebra and the exponential map. In this section we recall standard facts and notations from [19] (which as phrased below also holds for $p$-adic Lie groups). Let

$$
[\cdot, \cdot]: \mathfrak{g}^{2} \rightarrow \mathfrak{g}
$$

be the $\mathbb{Q}_{\sigma}$-bilinear Lie bracket satisfying

$$
\operatorname{Ad}_{g}([u, v])=\left[\operatorname{Ad}_{g} u, \operatorname{Ad}_{g} v\right]
$$

for all $g \in G$ and $u, v \in \mathfrak{g}$. Since $\mathbb{Q}_{\sigma}$ is a local field of characteristic zero, the exponential map $\exp (\cdot)$ is a local homeomorphism between the Lie algebra $\mathfrak{g}$ and $G$ such that

$$
g \exp (u) g^{-1}=\exp \left(\operatorname{Ad}_{g}(u)\right)
$$

whenever both sides are defined. Write $\log (\cdot)$ for the locally defined inverse map. Furthermore, we define $\operatorname{ad}_{u}(v)=[u, v]$ for any $u, v \in \mathfrak{g}$. Then

$$
\operatorname{Ad}_{\exp u}=\exp \left(\operatorname{ad}_{u}\right)
$$

whenever both sides are defined, here the exponential map on the right is defined for sufficiently small endomorphisms of $\mathfrak{g}$.

Recall that

$$
\left[\mathfrak{u}^{\alpha}, \mathfrak{u}^{\beta}\right] \subset \mathfrak{u}^{\alpha+\beta}
$$

for any roots $\alpha, \beta$ (which follows easily from (3.1)). If $\alpha \in \Phi$ is a root, then the exponential map is actually a polynomial map and so can be uniquely extended to the whole of $\mathfrak{u}^{\alpha} \oplus \mathfrak{u}^{2 \alpha}$ such that (3.2) and (3.3) still hold. The image $U^{\alpha}=\exp \left(\mathfrak{u}^{\alpha} \oplus \mathfrak{u}^{2 \alpha}\right)$ is the unipotent subgroup mentioned earlier. More generally, let $\Psi \subset \Phi$ be a set of roots such that $(\Psi+\Psi) \cap \Phi \subset \Psi$ and $|\alpha(a)|>1$ for all $\alpha \in \Psi$ and some fixed $a \in A$. Then

$$
\mathfrak{u}^{\Psi}=\sum_{\alpha \in \Psi} \mathfrak{u}^{\alpha}
$$

is a Lie algebra, $\exp (\cdot)$ can be uniquely extended to $\mathfrak{u}^{\Psi}$ such that (3.2) holds, and $U^{\Psi}=\exp \mathfrak{u}^{\Psi}$ is a unipotent subgroup (that is generated by the subgroups $U^{\alpha}$ for $\left.\alpha \in \Psi\right)$. We say that a subgroup $U^{\prime} \subset$ $U^{\Psi}$ is connected if there exists a Lie subalgebra $\mathfrak{u}^{\prime} \subset \mathfrak{u}^{\Psi}$ such that $U^{\prime}=\exp \left(\mathfrak{u}^{\prime}\right)$. Note that in the real case this notion agrees with the subgroup being connected with respect to the Hausdorff topology and that in general it is equivalent to $U^{\prime}$ being Zariski closed (and Zariski connected). 
3.3. The image of iterates of $\operatorname{ad}_{v}$ for $v \in \mathfrak{u}^{\alpha}$. We now formulate and prove a corollary of the structure of semi-simple groups and their maximal $\mathbb{Q}_{\sigma}$-split subtori. This will be the most important use of the assumption that $A$ is maximal - the structure provided by this result is crucial to our approach. For any subset $R \subset V$ we define

$$
\mathfrak{u}^{R}=\bigoplus_{\alpha \in R} \mathfrak{u}^{\alpha} \subset \mathfrak{g} \text {. }
$$

Proposition 3.2. Let $A$ be a maximal $\mathbb{Q}_{\sigma}$-split torus in a semi-simple linear algebraic group $\mathbf{G}$ defined over a local field $\mathbb{Q}_{\sigma}$ of characteristic zero. Let $\alpha$ be a root, and let $v \in \mathfrak{u}^{\alpha}$. Then for any root $\beta$ with $(\beta, \alpha)>0$ and $s_{\alpha}(\beta)=\beta-m \alpha$ the restriction of $\operatorname{ad}_{v}^{m}$ to $\mathfrak{u}^{s_{\alpha}(\beta)}$ is injective.

Proof of Lemma 3.1 and Proposition 3.2. Let $v \in \mathfrak{u}^{\alpha}$ be nonzero. As $v$ is a nonzero unipotent element in the semi-simple part of the Lie algebra of $\mathbb{G}$, the Jacobson-Morozov theorem (see e.g. [13, III, Lemma 7 and Theorem 17]) implies that $v$ must be one element of an $\mathfrak{s l}_{2^{-}}$ triple, i.e. there exists some $h$ and $w$ so that the Lie algebra generated by $v, h, w$ is isomorphic to $\mathfrak{s l}_{2}$. We claim that we may assume that $h$ belongs to the Lie algebra of $A$.

To see the claim, let $h=h_{0}+h_{1}$ be a decomposition of $h$ into an element $h_{0} \in \mathfrak{g}^{0}$ and an element $h_{1}$ which belongs to the sum of the root spaces. By assumption $[h, v]=2 v$, so that $2 v=\left[h_{0}, v\right]+\left[h_{1}, v\right]$. However, by (3.4) this implies that $\left[h_{1}, v\right]=0$. We also have that $h=$ $[v, w]=h_{0}+h_{1} \in \operatorname{Im}(\operatorname{ad}(v))$. However, by (3.4) we see that the image $\operatorname{Im}(\operatorname{ad}(v))$ is invariant under the adjoint action of $A$, which implies that $h_{0} \in \operatorname{Im}(\operatorname{ad}(v))$. However, this implies again by the Jacobson-Morozov theorem that there exists some $w^{\prime}$ such that $\left(v, h_{0}, w^{\prime}\right)$ is an $\mathfrak{s l}_{2}$-triple. If $w^{\prime} \notin \mathfrak{u}^{-\alpha}$, then we can split $w^{\prime}$ into two components $w^{-\alpha}+w^{\prime \prime}$, with $w^{-\alpha} \in \mathfrak{u}^{-\alpha}$ and $w^{\prime \prime}$ belonging to the remaining weight spaces. Since

$$
\left[h_{0}, w^{\prime}\right]=-2 w^{\prime}=\left[h_{0}, w^{-\alpha}+w^{\prime \prime}\right]=-2\left(w^{-\alpha}+w^{\prime \prime}\right)
$$

we get by comparing components from different weight spaces $\left[h_{0}, w^{-\alpha}\right]=$ $-2 w^{-\alpha}$. Moreover, $\left[v, w^{\prime}\right]=h_{0}=\left[v, w^{-\alpha}+w^{\prime \prime}\right]$ which by comparing components also implies $\left[v, w^{-\alpha}\right]=h_{0}$. Therefore, $\left(v, h_{0}, w^{-\alpha}\right)$ is an $\mathfrak{s l}_{2}$-triple (and now one can show that actually $w^{\prime}=w^{-\alpha}$ ).

From the existence of the above $\mathfrak{s l}_{2}$-triple it follows that there exists an algebraic subgroup $\mathbf{L}$ defined over $\mathbb{Q}_{\sigma}$ (defined e.g. by the Zariskiclosure of the image of a neighborhood of 0 in the span of the $\mathfrak{s l}_{2}$-triple under exp) with the span of the $\mathfrak{s l}_{2}$-triple as its Lie algebra. The subgroup $\mathbf{L}$ contains the split torus $\mathbf{T}=\mathbf{L} \cap C_{\mathbf{G}}(A)$ whose Lie algebra is spanned by the element $h_{0}$. However, as $\mathbf{T}$ commutes with $A$ and 
is split, this implies that $\mathbf{T}\left(\mathbb{Q}_{\sigma}\right) \subseteq A$. To summarize and to simplify the notation, we have shown that there exists an $\mathfrak{s l}_{2}$-triple $(v, h, w)$ for which $h$ belongs to the Lie algebra of $A$ and $w \in \mathfrak{u}^{-\alpha}$.

If now $\beta \in \Phi$ is a root and $m \geq 0$ for which $\beta+\alpha, \beta-(m+1) \alpha \notin \Phi$ but $\beta-\alpha, \ldots, \beta-m \alpha \in \Phi$, then $\mathfrak{u}^{\{\beta, \ldots, \beta-m \alpha\}}$ is a finite-dimensional representation for $\mathbf{L}$ (resp. its Lie algebra). Hence $\left.\operatorname{ad}(h)\right|_{\beta}$ equals multiplication by some $k$ and $\left.\operatorname{ad}(h)\right|_{\beta-m \alpha}$ equals multiplication by $-k$, which then also has to equal $k-2 m$. It follows that $k=m=\frac{(\alpha, \beta)}{(\alpha, \alpha)}$. It follows that the weight of any root space $\mathfrak{u}^{\beta}$ with respect to the $\mathfrak{s l}_{2^{-}}$ representation equals a constant multiple of $(\alpha, \beta)$. In particular, if we take an element of $\mathbf{T}\left(\mathbb{Q}_{\sigma}\right)$ that does not generate a compact subgroup, then this element or its inverse will satisfy the claim in Lemma 3.1.

Let now $\beta \in \Phi$ be any root with $(\beta, \alpha)>0$. Let $m \geq 1$ be such that $s_{\alpha}(\beta)=\beta-m \alpha$. Then the weight of $\beta$ (resp. the weight of $s_{\alpha}(\beta)$ ) with respect to the $\mathfrak{s l}_{2}$-representation equals $m$ (resp. $-m$ ). Hence the theory of finite-dimensional $\mathfrak{s l}_{2}$-representation implies the proposition.

3.4. The $S$-algebraic group $G$ and the locally homogeneous spaces $X$. Recall from the introduction and from Section 2 that we may assume $\mathbb{G}$ is a $\mathbb{Q}$-almost simple algebraic $\mathbb{Q}$-group. Let $S$ be a finite set of places (finite primes and the symbol $\infty$ ), and define for every $\sigma \in S$ the group $G_{\sigma}=\mathbb{G}\left(\mathbb{Q}_{\sigma}\right)$. If $\mathbb{G}(\mathbb{R})$ is not compact, we require that $\infty \in S$. We also define $G=\mathbb{G}\left(\mathbb{Q}_{S}\right)=\prod_{\sigma \in S} G_{\sigma}$, and suppose that $\Gamma<G$ is an arithmetic lattice, i.e. a lattice commensurable with $\mathbb{G}\left(\mathcal{O}_{S}\right)$ as in the introduction.

The quotient $X=\Gamma \backslash G$ is a locally homogeneous space; let $d(\cdot, \cdot)$ be a left invariant metric on $G_{S}$, and denote the induced metric on $X$ also by $d(\cdot, \cdot)$. The group $G$ acts on $X$ by right translations, i.e. $g \cdot x=x g^{-1}$ for $g \in G, x \in X$.

For every $\sigma \in S$ we can write the algebraic group $\mathbb{G}$ considered as an algebraic groups over $\mathbb{Q}_{\sigma}$ as an almost direct product of $\mathbb{Q}_{\sigma}$-almost simple groups $\prod_{f \in F_{\sigma}} \mathbf{G}_{f}$. We define $F=\bigsqcup_{\sigma \in S} F_{\sigma}$, and if $f \in F_{\sigma}$ we shall say that $\sigma$ is the place attached to $f$; often, when it is clear which $f$ we discuss, $\sigma$ will be implicitly assumed to be the attached place to $f$. We also write $\mathfrak{g}_{f}$ for the Lie algebra of $\mathbf{G}_{f}$.

Let $F^{\prime} \subset F$ and fix for every $f \in F^{\prime}$ a maximal $\mathbb{Q}_{\sigma}$-split torus $\mathbf{A}_{f}$. Furthermore, we choose for every $f \in F^{\prime}$ a finite index subgroup $A_{f}$ of the group of $\mathbb{Q}_{\sigma}$-points of this torus. 
For a root $\alpha$ of $G_{f_{0}}$ for $f_{0} \in F^{\prime}$ we define an associated Lyapunov root to be the group homomorphism $A \rightarrow \mathbb{R}^{+}$given by

$$
a=\left(\left(a_{f}\right)_{f \in F^{\prime}}\right) \mapsto \log \left|\alpha\left(a_{f_{0}}\right)\right|_{\sigma_{0}},
$$

where $\sigma_{0}$ is the place corresponding to $f_{0}$ and the absolute value is taken in $\mathbb{Q}_{\sigma_{0}}$. We write $\Phi_{f_{0}}$ for the set of Lyapunov roots of $G_{f_{0}}$.

We denote by $\Phi^{\prime}=\bigcup_{f \in F^{\prime}} \Phi_{f}$ the set of all Lyapunov roots obtained in this way and will continue to use Greek letters to denote Lyapunov roots - in fact we will not distinguish between the root and its associated Lyapunov root. As discussed in Section 3.1 for every $f \in F^{\prime}$ the Lyapunov roots for $G_{f}$ span a vector space $V_{f}$ over $\mathbb{R}$ with a natural Euclidean inner product which is preserved by the Weyl group; we define the inner product on the product of these Euclidean spaces so that the individual subspaces $V_{f}$ for $f \in F^{\prime}$ are orthogonal. The orthogonal reflections $s_{\alpha}$ for $\alpha \in \Phi_{F^{\prime}}$ are naturally extended to all of $V$, acting as the identity on the orthogonal complement of the subspace where $s_{\alpha}$ was originally defined.

For a Lyapunov root $\alpha \in \Phi_{f}$ we will write $U^{\alpha}$ and $\mathfrak{u}^{\alpha}$ for the subgroup of $G_{f}$ resp. the Lie subalgebra of $\mathfrak{g}_{f}$ corresponding to the root as in Section 3.1. Furthermore, we define $\mathfrak{g}^{0}=\sum_{f \in F^{\prime}} \mathfrak{g}_{f}^{0}+\sum_{f \in F \backslash F^{\prime}} \mathfrak{g}_{f}$ (which has the structure of an additive group, but for $|S| \geq 2$ is no longer a Lie algebra in the usual sense).

Finally we say a subgroup $U<G_{S}$ is unipotent and connected if $U$ is a direct product of unipotent (Zariski) connected subgroups $U_{\sigma}<G_{\sigma}$ for $\sigma \in S$. For instance we can define for the subset $\Psi=\left\{\alpha \in \Phi^{\prime}\right.$ : $\alpha(a)<0\}$ the connected unipotent subgroup

$$
U^{\Psi}=\prod_{f \in F^{\prime}} U^{\Psi \cap \Phi_{f}}
$$

where $U^{\Psi \cap \Phi_{f}}<G_{f}$ is the unipotent subgroup over $\mathbb{Q}_{\sigma}$ associated to all roots that are contracted by $a_{f}$. It will also be convenient to notice the following simple fact: any closed subgroup $U<U^{\Psi}$ is automatically a product of unipotent subgroups $U_{\sigma}$ for $\sigma \in S$. If $U$ is additionally $A$-normalized, then $U$ is moreover a product of unipotent subgroups $U_{f}$ for $f \in F^{\prime}$.

\section{LEAF-WISE MEASURES AND ENTROPY}

Leafwise measures (which are also referred to as conditional measures on leaves of a foliation) were used in this context the first time by Katok and Spatzier in their pioneering work [17], though implicitly they can also be found hidden in the arguments Rudolph's original paper on the subject [35]. A fairly general construction of leafwise measures 
is presented in [20, Sect. 3]. A self-contained and comprehensive construction of these measures (though somewhat less general, as it only treats the case of foliations whose leaves are given by orbits of groups) as well as the relation of these leafwise measures to entropy is given in [9]. Here we only summarize without proofs the main properties of these leafwise measures.

4.1. Basic properties. We will be working with connected subgroups of $U^{\Psi}$ for $\Psi \subset \Phi^{\prime}$ with $(\Psi+\Psi) \cap \Phi^{\prime} \subset \Psi$ and $|\alpha(a)|<1$ for some fixed $a \in A$ and all $\alpha \in \Psi$. These subgroups are automatically unipotent. If $U<G_{S}$ is normalized by $A$ then for every $x \in X$ and $a \in A$, $a(U . x)=x U a^{-1}=x a^{-1} U=U . a(x)$, so that the foliation of $X$ into $U$-orbits is invariant under the action of $A$. We will say that $a \in A$ expands (contracts) the $U$-leaves, or simply $U$, if the absolute values of all eigenvalues of $\operatorname{Ad}_{a}$ restricted to the Lie algebra of $U$ are greater (smaller) than one.

For any locally compact metric space $Y$ let $\mathcal{M}_{\infty}(Y)$ denote the space of Radon measures on $Y$ equipped with the weak* topology, i.e. all locally finite Borel measures on $Y$ with the coarsest topology for which $\rho \mapsto \int_{Y} f(y) \mathrm{d} \rho(y)$ is continuous for every compactly supported continuous $f$. For two Radon measures $\nu_{1}$ and $\nu_{2}$ on $Y$ we write

$$
\nu_{1} \propto \nu_{2} \text { if } \nu_{1}=C \nu_{2} \text { for some } C>0
$$

and say that $\nu_{1}$ and $\nu_{2}$ are proportional.

We let $B_{\epsilon}^{Y}(y)$ (or $B_{\epsilon}(y)$ if $Y$ is understood) denote the ball of radius $\epsilon$ around $y \in Y$; if $H$ is a group we set $B_{\epsilon}^{H}=B_{\epsilon}^{H}(e)$; and if $H$ acts on $X$ and $x \in X$ we let $B_{\epsilon}^{H}(x)=B_{\epsilon}^{H} . x$.

Let $\mu$ be an $A$-invariant probability measure on $X$. For any unipotent subgroup $U<U^{\Psi}<G_{S}$ normalized by $A$, one has a system $\left\{\mu_{x, U}\right\}_{x \in X}$ of Radon measures on $U$ and a co-null set $X^{\prime} \subset X$ with the following properties:

(1) The map $x \mapsto \mu_{x, U}$ is measurable.

(2) For every $\epsilon>0$ and $x \in X^{\prime}$ it holds that $\mu_{x, U}\left(B_{\epsilon}^{U}\right)>0$.

(3) For every $x \in X^{\prime}$ and $u \in U$ with $u . x \in X^{\prime}$, we have that $\mu_{x, U} \propto\left(\mu_{u . x, U}\right) u$, where $\left(\mu_{u . x, U}\right) u$ denotes the push forward of the measure $\mu_{u \cdot x, U}$ under the map $v \mapsto v u$.

(4) For every $a \in A$, and $x, a(x) \in X^{\prime}, \mu_{a(x), U} \propto a\left(\mu_{x, U}\right) a^{-1}$.

In general, there is no canonical way to normalize the measures $\mu_{x, U}$; we fix a specific normalization by requiring that $\mu_{x, U}\left(B_{1}^{U}\right)=1$ for every $x \in X^{\prime}$. This implies the next crucial property.

(5) If $U \subset C(a)=\left\{g \in G_{S}: g a=a g\right\}$ is centralized by $a \in A$, then $\mu_{a(x), U}=\mu_{x, U}$ whenever $x, a(x) \in X^{\prime}$. 
(6) $\mu$ is $U$-invariant if, and only if, $\mu_{x, U}$ is a Haar measure on $U$ a.e. (see [20, Prop. 4.3]).

The other extreme to invariance as above is where $\mu_{x, U}$ is atomic. If $\mu$ is $A$-invariant then outside some set of measure zero if $\mu_{x, U}$ is atomic then it is supported on the single point $e \in U$, in which case we say that $\mu_{x, U}$ is trivial. The leafwise measures for the unipotent subgroup $U^{\alpha}$ associated to a Lyapunov root $\alpha \in \Phi^{\prime}$ we denote by $\mu_{x}^{\alpha}$, and more generally we write $\mu_{x}^{\Psi}$ for the leafwise measures on $U^{\Psi}$ when $\Psi \subset \Phi^{\prime}$ is a set of Lyapunov roots such that $U^{\Psi}$ is a unipotent subgroup.

Fundamental to us is the following characterization of positive entropy for the action of an element $a \in A$ on an $A$-invariant and ergodic probability measure $\mu$

(7) Let $\Psi=\left\{\alpha \in \Phi^{\prime}: \alpha(a)<0\right\}$ so that $U^{\Psi}$ is the horospherical stable subgroup defined by $a$. Then the measure theoretic entropy $\mathrm{h}_{\mu}(a)$ is positive if and only if the leafwise measures $\mu_{x}^{\Psi}$ are nonatomic a.e.

Hence positive entropy implies that certain leafwise measures are nontrivial a.e.

4.2. Entropy contribution. In this section we refine property (7) from above to a more quantitative statement, for more details see [24, Sect. 9], [4, Sect. 9] or [9, Sect. 7].

Let $U$ be a connected subgroup normalized by $A$ such that $a \in A$ contracts $U$. We let

$$
\theta_{a}(g)=a g a^{-1}
$$

be the inner automorphism defined by $a$. Then for any $a$-invariant probability measure $\mu$ on $X$ the limit

$$
\operatorname{vol}_{\mu}(a, U, x)=-\lim _{n \rightarrow \infty} \frac{\log \mu_{x}^{U}\left(\theta_{a}^{n}\left(B_{1}^{U}\right)\right)}{n}
$$

exists for a.e. $x \in X$ by [4, Lemma 9.1]. If furthermore, $\mu_{x}^{U}$ is supported by a subgroup $P \subseteq U$ that is connected and normalized by $A$, then

$$
\operatorname{vol}_{\mu}(a, U, x) \leq \bmod (a, P)=\sum_{\alpha \in \Phi^{\prime}} \alpha(a)^{-} \operatorname{dim}_{\mathbb{Q}_{\sigma(\alpha)}}\left(\mathfrak{p} \cap \mathfrak{u}^{\alpha}\right)
$$

for a.e. $x \in X$. Here we write $r^{-}=\max (0,-r)$ for the negative part of $r \in \mathbb{R}, \mathfrak{p}$ for the Lie algebra of $P$ and $\mathbb{Q}_{\sigma(\alpha)}$ denotes the field over which the Lyapunov root $\alpha \in \Phi^{\prime}$ is defined. Note that $\mathfrak{p}$ is a direct sum of its subspaces $\mathfrak{p} \cap \mathfrak{u}^{\alpha}$, since $P$ is normalized by $A$. In fact, $\bmod (a, P)$ is the negative logarithm of the module of the restriction of $\theta_{a}$ to $P$. It is easy to check that $\operatorname{vol}_{\mu}(a, U, \cdot)$ is $A$-invariant, and so constant for an 
$A$-ergodic measure. We write $\mathrm{h}_{\mu}(a, U)$ for the integral of $\operatorname{vol}_{\mu}(a, U, \cdot)$, and will refer to it as the entropy contribution of $U$.

In the case where $U=U^{\alpha}<G_{f}$ for $\alpha \in \Phi_{f}$ as in Section 3.2, the map $a \mapsto \operatorname{vol}_{\mu}(a, U, x)$ restricted to the half space $\alpha(a) \leq 0$ can be extended to a homomorphism of groups from $A$ to the additive group on $\mathbb{R}$. To see this, note first that $\theta_{a}^{n}\left(B_{1}^{U}\right)$ only depends on the value of $n \alpha(a)$ and moreover, $\theta_{a}^{n}\left(B_{1}^{U}\right) \subset \theta_{a}^{m}\left(B_{1}^{U}\right)$ whenever $n \alpha(a)<m \alpha(a)<0$. Therefore, there exists $\delta_{\alpha} \geq 0$ with

$$
\mathrm{h}_{\mu}\left(a, U^{\alpha}\right)=\delta_{\mu}^{\alpha} \alpha(a)^{-} \text {for all } a \in A \text { with } \alpha(a) \leq 0,
$$

we refer to $\delta_{\mu}^{\alpha}$ as the dimension of $\mu$ along $U^{\alpha}$. Now (4.2) is equivalent to

$$
\delta_{\mu}^{\alpha} \leq \operatorname{dim}_{\mathbb{Q}_{\sigma}}\left(\mathfrak{p} \cap \mathfrak{u}^{\alpha}\right)+2 \operatorname{dim}_{\mathbb{Q}_{\sigma}}\left(\mathfrak{p} \cap \mathfrak{u}^{2 \alpha}\right),
$$

where we agree to set the second term to zero if $2 \alpha$ is not a root.

A $\sigma$-algebra $\mathcal{A}$ of Borel subsets of $X$ is subordinate to $U$ if $\mathcal{A}$ is countably generated, for every $x \in X$ the atom $[x]_{\mathcal{A}}$ of $x$ with respect to $\mathcal{A}$ is contained in the leaf $U x$, and for a.e. $x$

$$
B_{\epsilon}^{U} x \subseteq[x]_{\mathcal{A}} \subseteq B_{\rho}^{U} x \text { for some } \epsilon>0 \text { and } \rho>0 .
$$

A $\sigma$-algebra $\mathcal{A}$ is a-decreasing if $a^{-1} \mathcal{A} \subseteq \mathcal{A}$. By [4, Lemma 9.3] we have

$$
\mathrm{H}_{\mu}\left(\mathcal{A} \mid a^{-1} \mathcal{A}\right)=\int \operatorname{vol}_{\mu}(a, U, x) \mathrm{d} \mu .
$$

whenever $\mathcal{A}$ is an $a$-decreasing $\sigma$-algebra that is subordinate to $U$.

It has been shown in [24, Prop. 9.2] that there exists an $a$-decreasing $\sigma$-algebra $\mathcal{A}$ subordinate to $U$ provided $\mu$ is $a$-ergodic. Moreover, if $U$ is the horospherical stable subgroup defined by $a$, then $\mathrm{h}_{\mu}(a)=$ $\mathrm{H}_{\mu}\left(\mathcal{A} \mid a^{-1} \mathcal{A}\right)$. This can be used to show that the entropy equals the entropy contribution of the horospherical stable subgroup

$$
\mathrm{h}_{\mu}(a)=\int \operatorname{vol}_{\mu}(a, U, x) \mathrm{d} \mu
$$

even if $\mu$ in not $a$-ergodic, see [4, Prop. 9.4].

4.3. The structure of leafwise measures for the horospherical subgroup. We recall and slightly simplify [4, Thm. 8.4-8.5], which describe the structure of the leafwise measure $\mu_{x}^{U}$ for the horospherical stable subgroup $U$ defined by $a$. Under the assumption that $\mu$ is an $A$ ergodic and invariant probability measure on $X$ these theorems simplify to the following statements due to the fact that the adjoint action restricted to $A$ has its eigenvalues in $\mathbb{Q}_{\sigma}$.

Let $\Psi=\left\{\alpha \in \Phi^{\prime}: U^{\alpha} \subset U\right.$ and $\alpha$ is indivisible $\}$. Fix some order of the elements of $\Psi=\left\{\alpha_{1}, \ldots, \alpha_{\ell}\right\}$ and define $\phi: \prod_{i=1}^{\ell} U^{\alpha_{i}} \rightarrow U$ by 
$\phi\left(u_{1}, \ldots, u_{\ell}\right)=u_{1} \cdots u_{\ell}$ for any $\left(u_{1}, \ldots, u_{\ell}\right) \in \prod_{i=1}^{\ell} U^{\alpha_{i}}$. Here $U^{\alpha}=$ $\exp \left(\mathfrak{u}^{\alpha} \oplus \mathfrak{u}^{2 \alpha}\right)$; in the terminology of [4, Sect. 4.4] these are precisely the coarse Lyapunov subgroups of the action of $A$. Therefore, [4, Thm. 8.4] gives

$$
\mu_{x}^{U} \propto \phi_{*}\left(\mu_{x}^{\alpha_{1}} \times \cdots \times \mu_{x}^{\alpha_{\ell}}\right) \text { a.e. }
$$

This and (4.1) readily implies that the entropy

$$
\mathrm{h}_{\mu}(a)=\sum_{i=1}^{\ell} \mathrm{h}_{\mu}\left(a, U^{\alpha_{i}}\right)
$$

is the sum over all entropy contributions of $U^{\alpha} \subset U$. In particular, positive entropy implies that there exists an indivisible root $\alpha$ so that the leafwise measure $\mu_{x}^{\alpha}$ is nontrivial a.e. - indeed, so that the dimension $\delta_{\mu}^{\alpha}$ of $\mu$ along $U^{\alpha}$ is positive.

The following is a version of the high entropy theorem which is basically contained in [4] and generalizes [3]; see also [9, Sect. 9] for a simplified derivation of this result:

Theorem 4.1. Let $\mu$ be an A-invariant and ergodic probability measure on $X=\Gamma \backslash G_{S}$. Let $a \in A$ with stable horosperical subgroup $U=U_{a}$. Then there exist two connected A-normalized subgroups $H<P<U$ such that

(1) $\mu_{x}^{U}$ is supported by $P$ a.e.

(2) $\mu_{x}^{U}$ is left- and right-invariant under multiplication with elements of $H$.

(3) $H$ is a normal subgroup of $P$ and any elements $g \in P \cap U^{\alpha_{r}}$ and $h \in P \cap U^{\alpha_{s}}$ of different root subgroups $(r \neq s)$ satisfy that $g H$ and $h H$ commute with each other in $P / H$.

(4) $\mu_{x}^{\alpha_{i}}$ is left- and right-invariant under multiplication with elements of $H \cap U^{\alpha_{i}}$ for $i=1, \ldots, \ell$.

Proof. By [4, Thm. 8.5] there exist for a.e. $x \in X$ two subgroups $H_{x}<P_{x}<U$ with properties (1)-(4) of the theorem at the point $x$. Additionally, these subgroups are connected and their Lie algebras are direct sums of subspaces of root spaces $\mathfrak{u}^{\alpha}$ - in the notation of [4, Thm. 8.5] this is the same as $H_{x}$ and $P_{x}$ allowing a weight decomposition. However, in our case this implies that these subgroups are normalized by $A$ (this would not necessarily be so if we were working over field extensions of $\mathbb{Q}_{\sigma}$ ). This together with Property (4) of $\S 4.1$ shows that for a.e. $x \in X$ the subgroups $H_{x}<P_{x}$ also satisfies Properties (1)-(4) at $a x$ for any $a \in A$. Using ergodicity of $\mu$ it is not difficult to find subgroups $H$ and $P$ as in the theorem, see for instance [6, Lemma 3.5] where this has been done for $P$. 
For the Lie algebras $\mathfrak{h} \subset \mathfrak{p} \subset \mathfrak{u}$ of $H<P<U$ we get the following corollary. This actually appears in the proof of [4, Thm. 8.5], but is also immediate from Theorem 4.1 and the Campbell-Hausdorff formula.

Corollary 4.2. For any two linearly independent roots $\alpha, \beta$ with $\mathfrak{u}^{\alpha}, \mathfrak{u}^{\beta}<$ $U$ we have $\left[\mathfrak{p} \cap \mathfrak{u}^{\alpha}, \mathfrak{p} \cap \mathfrak{u}^{\beta}\right] \subset \mathfrak{h} \cap \mathfrak{u}^{\alpha+\beta}$.

While the subgroup $P$ gives an upper bound for the entropy contribution as we have seen in $\S 4.2$, the subgroup $H$ gives a lower bound as follows.

Lemma 4.3. Let $H<U$ be connected subgroups normalized by $A$ such that $a \in A$ contracts $U$. If $\mu$ is invariant under $H$, then

$$
\mathrm{h}_{\mu}(a, U) \geq \sum_{\alpha \in \Psi} \alpha(a)^{-} \operatorname{dim}_{\mathbb{Q}_{\sigma}}\left(\mathfrak{h} \cap \mathfrak{u}^{\alpha}\right),
$$

where $\mathfrak{h}$ is the Lie algebra of $H$ and $\Psi$ is the set of roots with $\alpha(a)<0$.

The lemma follows from the following two observations that only rely on the definitions: If $H<U$ then $\mathrm{h}_{\mu}(a, H) \leq \mathrm{h}_{\mu}(a, U)$. If $\mu$ is $H$ invariant, then $\mu_{x}^{H}$ is the Haar measure of $H$ for a.e. $x$ and $\mathrm{h}_{\mu}(a, H)=$ $\bmod (a, H)$ is the negative logarithm of the module of conjugation by $a$ restricted to $H$.

\section{THE HIGH ENTROPY ROOTS}

It will be convenient to divide the indivisible roots in $\Phi^{\prime}$ into three classes depending on the measure $\mu$ :

(1) the roots with no attached entropy, i.e. for which $\delta_{\mu}^{\alpha}=0$

(2) the roots $\alpha \in \Phi^{\prime}$ for which $\delta_{\mu}^{\alpha}>0$ but $\delta_{\mu}^{\beta}=0$ for every indivisible root $\beta \neq \alpha$ with $(\alpha, \beta) \neq 0$

(3) the roots $\alpha \in \Phi^{\prime}$ for which $\delta_{\mu}^{\alpha}>0$ and also $\delta_{\mu}^{\beta}>0$ for some indivisible $\beta \neq \alpha$ with $(\alpha, \beta) \neq 0$.

In the second case we say that $\alpha$ is a root of low entropy, and in the third $\alpha$ is a root of high entropy.

In this section we deal with the high entropy roots, and show in particular that if $\alpha$ is a root of high entropy then $\mu$ is invariant under a subgroup generated by unipotent elements. We also establish a symmetric property of the $\delta_{\mu}^{\alpha}$, namely that $\delta_{\mu}^{\alpha}$ is positive if and only if $\delta_{\mu}^{-\alpha}$ is.

Theorem 5.1. Let $\mu$ be an A-invariant and ergodic probability measure on $X=\Gamma \backslash G$. Then the dimensions of $\mu$ along the subgroups associated to the roots are symmetric, i.e. $\delta_{\mu}^{\alpha}=\delta_{\mu}^{-\alpha}$ for any indivisible $\alpha \in \Phi$. Moreover, if $\alpha$ is a high entropy root for $\mu$, then $\mu$ is invariant under a 
nontrivial subgroup of $U^{\alpha}$; in fact, under the unipotent group $P^{\alpha}<U^{\alpha}$ of Theorem 4.1, which is nontrivial as $\delta_{\mu}^{\alpha}>0$.

Proof. We first prove $\delta_{\mu}^{\alpha} \leq \delta_{\mu}^{-\alpha}$ for an arbitrary indivisible (Lyapunov) root $\alpha$, which implies the symmetry. If $\delta_{\mu}^{\alpha}=0$ this is trivial. So suppose $\delta_{\mu}^{\alpha}>0$ and choose $a_{\alpha} \in A$ as in Lemma 3.1 so that $\beta\left(a_{\alpha}\right)=c(\alpha, \beta)$ for all Lyapunov roots $\beta$ and some $c>0$. By (4.6) and (4.3) we can express the entropy of $a_{\alpha}$ as

$$
\mathrm{h}_{\mu}\left(a_{\alpha}\right)=c \sum_{\beta:(\alpha, \beta)<0} \delta_{\mu}^{\beta}(\alpha, \beta)^{-} .
$$

Since $\mathrm{h}_{\mu}\left(a_{\alpha}\right)=\mathrm{h}_{\mu}\left(a_{\alpha}^{-1}\right)$ we also have

$$
\mathrm{h}_{\mu}\left(a_{\alpha}\right)=c \sum_{\beta:(\alpha, \beta)>0} \delta_{\mu}^{\beta}(\alpha, \beta)^{+} .
$$

However, $s_{\alpha}: \Phi^{\prime} \rightarrow \Phi^{\prime}$ maps all the roots $\beta$ appearing in (5.1) to those appearing in the second sum without changing the absolute value of the inner products, and so the last formula can also be written as

$$
\mathrm{h}_{\mu}\left(a_{\alpha}\right)=c \sum_{\beta:(\alpha, \beta)<0} \delta_{\mu}^{s_{\alpha}(\beta)}(\alpha, \beta)^{-} .
$$

We claim that $\delta_{\mu}^{\beta} \leq \delta_{\mu}^{s_{\alpha}(\beta)}$ for any $\operatorname{root} \beta \notin\langle\alpha\rangle$ with $(\alpha, \beta)<0$. It is clear that this together with (5.1)-(5.2) implies $\delta_{\mu}^{\alpha} \leq \delta_{\mu}^{-\alpha}$.

For the proof of the claim fix $\beta$ and choose $a \in A$ such that

$$
\alpha(a), \beta(a)<0,
$$

which is possible since $\alpha, \beta$ are linearly independent. Let $U$ be the stable horospherical subgroup defined by a so that $U^{\alpha}, U^{\beta}<U$. Recall that a.e. $\mu_{x}^{U}$ is the product measure of all the leafwise measures associated to indivisible roots whose subgroups are contained in $U$. Now let $H<P<U$ be as in Theorem 4.1 so that $P$ supports $\mu_{x}^{U}$ a.e. and both subgroups are normalized by $A$. Then $P \cap U^{\alpha}$ supports $\mu_{x}^{\alpha}$ a.e. Since $\delta_{\mu}^{\alpha}>0$ these leafwise measures are nontrivial a.e. and there exists a nonzero $v \in \mathfrak{p} \cap \mathfrak{u}^{\alpha^{\prime}}$ where $\alpha^{\prime}$ is either $\alpha$ or $2 \alpha$. Note that $s_{\alpha^{\prime}}=s_{\alpha}$. Let $m$ be such that $s_{\alpha^{\prime}}(\beta)=\beta+m \alpha^{\prime}$. Define $V=\mathfrak{p} \cap \mathfrak{u}^{\beta}$, then Corollary 3.2 shows that $\operatorname{ad}_{v}^{m}$ maps $V$ to a subspace of $\mathfrak{u}^{s_{\alpha}(\beta)}$ of the same dimension. By Corollary 4.2 we have $\operatorname{ad}_{v}^{m}(V) \subset \mathfrak{h} \cap \mathfrak{u}^{s_{\alpha}(\beta)}$, hence

$$
\operatorname{dim}_{\mathbb{Q}_{\sigma}} \mathfrak{p} \cap \mathfrak{u}^{\beta} \leq \operatorname{dim}_{\mathbb{Q}_{\sigma}} \mathfrak{h} \cap \mathfrak{u}^{s_{\alpha}(\beta)} .
$$

The same argument also shows that

$$
\operatorname{dim}_{\mathbb{Q}_{\sigma}} \mathfrak{p} \cap \mathfrak{u}^{2 \beta} \leq \operatorname{dim}_{\mathbb{Q}_{\sigma}} \mathfrak{h} \cap \mathfrak{u}^{2 s_{\alpha}(\beta)} .
$$


Lemma 4.3 and (4.2) now imply for an indivisible root $\beta \neq \alpha$ with $(\alpha, \beta)<0$ that

$$
\delta_{\mu}^{s_{\alpha}(\beta)} \geq \operatorname{dim}_{\mathbb{Q}_{\sigma}} \mathfrak{p} \cap \mathfrak{u}^{\beta}+2 \operatorname{dim}_{\mathbb{Q}_{\sigma}} \mathfrak{p} \cap \mathfrak{u}^{2 \beta} \geq \delta_{\mu}^{\beta}
$$

as claimed. This implies the symmetry of the dimensions $\delta_{\mu}^{\alpha}=\delta_{\mu}^{-\alpha}$ for all indivisible roots $\alpha$.

Suppose now $\beta$ is a high entropy root and $\alpha \in \Phi$ a distinct indivisible root with $(\alpha, \beta) \neq 0$ and $\delta_{\mu}^{\alpha}>0$. We claim that

$$
\mathfrak{h} \cap\left(\mathfrak{u}^{\beta} \oplus \mathfrak{u}^{2 \beta}\right)=\mathfrak{p} \cap\left(\mathfrak{u}^{\beta} \oplus \mathfrak{u}^{2 \beta}\right)
$$

from which it follows that $H^{\beta}=P^{\beta}$ i.e. $\mu$ is $P^{\beta}$-invariant. By the symmetry properties of $\delta_{\mu}^{\alpha}$, if $\beta$ is a high entropy root so is $-\beta$, hence $\mu$ will also be $P^{-\beta}$-invariant and the theorem follows.

It remains to establish (5.4). By symmetry of the dimensions we may assume that $(\alpha, \beta)<0$. Then (5.3) implies

$$
\operatorname{dim}_{\mathbb{Q}_{\sigma}} \mathfrak{h} \cap \mathfrak{u}^{s_{\alpha}(\beta)} \geq \operatorname{dim}_{\mathbb{Q}_{\sigma}} \mathfrak{p} \cap \mathfrak{u}^{\beta} \geq \operatorname{dim}_{\mathbb{Q}_{\sigma}} \mathfrak{h} \cap \mathfrak{u}^{\beta}
$$

and similary for $2 \beta$. Also by symmetry we may apply the above to $-\alpha, \beta$ to get

$$
\operatorname{dim}_{\mathbb{Q}_{\sigma}} \mathfrak{h} \cap \mathfrak{u}^{\beta} \geq \operatorname{dim}_{\mathbb{Q}_{\sigma}} \mathfrak{p} \cap \mathfrak{u}^{s_{\alpha}(\beta)} \geq \operatorname{dim}_{\mathbb{Q}_{\sigma}} \mathfrak{h} \cap \mathfrak{u}^{s_{\alpha}(\beta)},
$$

and similarly for $2 \beta$. Together these imply (5.4).

\section{THE LOW ENTROPY ROOTS}

To deal with the low entropy root, we need to apply the main theorem of [8]. In order to state this theorem, we first need to introduce some terminology.

Let $X$ be a locally compact metric space with a Borel probability measure $\mu$, let $a: X \rightarrow X$ be a measure preserving transformation of $(X, \mu)$, let $H$ be a locally compact metric group acting continuously and locally free on $X$. We denote the action by $h . x$ for $h \in H$ and $x \in X$. Let $F: X \rightarrow Y$ be a measurable map to a Borel space $Y$. We say $\mu$ is $(F, H)$-transient if there exists a set $X^{\prime} \subset X$ of full measure such that there are no two different $x, y \in X^{\prime}$ on the same $H$-orbit $H . x=H . y$ with $F(x)=F(y)$. We say $\mu$ is $(F, H)$-exceptional if for every $\epsilon>0$ and compact neighborhood $O$ of the identity in $H$ there exists $B \subset X$ with $\mu(B)>1-\epsilon$ and some $\delta>0$ such that $x, y \in B$ with distance $d(x, y)<\delta$ and $F(x)=F(y)$ implies that $y=h . x$ for some $h \in O$.

We fix some semi-simple $a \in G$ that is diagonalizable over the ground field $\mathbb{R}$ resp. $\mathbb{Q}_{p}$. More precisely, we require that the adjoint representation is diagonalizable over the ground field and that there are no 
two distinct eigenvalues for $\operatorname{Ad} a$ with the same absolute value, and in particular that 1 is the only eigenvalue of absolute value one. We assume that $a$ uniformly contracts the leaves of the foliation defined by the $U$-orbits of some nontrivial closed unipotent subgroup $U<G$. In other words that $a$ normalizes $U$ and $a^{n} u a^{-n} \rightarrow e$ for $n \rightarrow \infty$ and all $u \in U$. We assume that $U$ is a direct product of Zariski closed unipotent subgroups $U_{\sigma}$ of $G_{\sigma}$ for $\sigma \in S$.

We are going to use the following abbreviations for the stable respectively unstable horospherical subgroup

$$
\begin{aligned}
& G^{-}=\left\{g \in G: a^{n} g a^{-n} \rightarrow e \text { for } n \rightarrow \infty\right\}, \\
& G^{+}=\left\{g \in G: a^{n} g a^{-n} \rightarrow e \text { for } n \rightarrow-\infty\right\},
\end{aligned}
$$

and the central subgroup

$$
G^{0}=\{g \in G: a g=g a\} .
$$

Recall that recurrence of $U$ with respect to $\mu$ is equivalent to $\mu_{x}^{U}(U)=$ $\infty$ by [20, Prop. 4.1]. We say $\mu$ is faithfully $U$-recurrent if for almost every $x$ there does not exist a Zariski closed proper subgroup $U^{\prime}<U$ such that $\mu_{x}^{U}$ is supported on $U^{\prime}$ (in other words we have $P=U$ for the subgroup $P$ as in Theorem 4.1). In [8] we have shown the following dichotomy for $\mu$ holds in the low entropy case $^{5}$ :

Theorem 6.1. Suppose $X=\Gamma \backslash G, a \in G$, and $U<G$ are as above, with $G$ an $S$-algebraic linear group. Assume that

- for every $\sigma \in S$ with $a_{\sigma} \neq e$ there is a homomorphism $\phi_{\sigma}$ : $\mathrm{SL}_{2}\left(\mathbb{K}_{\sigma}\right) \rightarrow G_{\sigma}$ such that $a_{\sigma}=\phi_{\sigma}\left(\left(\begin{array}{cc}t & \\ & t^{-1}\end{array}\right)\right)$ for some $t \in \mathbb{K}_{\sigma}^{\times}$ with $\|t\|_{\sigma}<1$ and $\phi_{\sigma}\left(\left(\begin{array}{ll}1 & * \\ & 1\end{array}\right)\right) \subset U_{\sigma}$.

Then a-invariant and faithfully $U$-recurrent probability measure $\mu$ on $X$ satisfies at least one of the following conditions:

(1) $\mu$ is $\operatorname{not}\left(\mu_{x}^{U}, C_{G}(U) \cap G^{-}\right)$-transient.

(2) $\mu$ is $\left(\mu_{x}^{U}, C_{G}(U)\right)$-exceptional.

In the context of the proof of Theorem 1.1 the above gives us the following corollary.

Corollary 6.2. Let $X=\Gamma \backslash G, A$, and $\mu$ be as in Theorem 1.1. Suppose $\mu$ has low entropy for some root $\alpha \in \Psi$. Let $P^{\alpha} \subset U^{\alpha}\left(P^{-\alpha} \subset U^{-\alpha}\right)$ be

\footnotetext{
${ }^{5}$ In fact, the main result of [8] is slightly more general than what is quoted above. Note also that in Theorem 6.1 no assumptions are made regarding the characteristic of the global field $\mathbb{K}$; we plan to explore positive characteristic analogues of our main theorem elsewhere.
} 
the minimal connected subgroup for which $\mu_{x}^{\alpha}$ is supported on $P^{\alpha}\left(\mu_{x}^{-\alpha}\right.$ is supported on $P^{-\alpha}$ ) for a.e. $x$. Then one of the following conditions must hold:

(1) (Invariance) $\mu$ is invariant under a nontrivial connected subgroup $H^{\alpha} \subset P^{\alpha}$ or under a nontrivial connected subgroup $H^{-\alpha} \subset P^{-\alpha}$.

(2) (Exceptional) $\mu$-a.e. ergodic component $\mu_{x}^{\mathcal{E}}$ for the action of $A^{\prime}=$ $\left\{a^{\prime} \in A \mid \alpha\left(a^{\prime}\right)=0\right\}$ is concentrated on a single orbit of $C_{G}\left(P^{\alpha}\right) \cap$ $C_{G}\left(P^{-\alpha}\right) \subset C_{G}\left(a_{\alpha}\right)$.

Here $\mathcal{E}$ equals the $\sigma$-algebra that consists of $A^{\prime}$-invariant sets. With this definition the conditional measures $\mu_{x}^{\mathcal{E}}$ give indeed the $A^{\prime}$-ergodic components of $\mu$. We say that a probability measure $\nu$ is concentrated on a set $B \subset X$ if $\nu(B)=1$. With some more effort, it is possible to deduce that if (2) does not hold, both $H^{\alpha}$ and $H^{-\alpha}$ are non-trivial (cf. [5, Sect. 4.1]), though for our purposes the weaker conclusion given above suffice.

Proof of Corollary 6.2. Let $\alpha \in \Phi^{\prime}$ be as in the corollary. By Theorem 5.1 it follows that $\delta_{\mu}^{\alpha}=\delta_{\mu}^{-\alpha}>0$. Essentially by construction, the subgroup $P^{\alpha}$ satisfies that $\mu$ is faithfully $P^{\alpha}$-recurrent. Indeed, if on a set of positive measure there would exists smaller subgroups on which $\mu_{x}^{P^{\alpha}}=\mu_{x}^{\alpha}$ is supported, then the fact that $A$ acts as multiplication by some elements of $\mathbb{Q}_{\sigma}$ on the Lie algebra of $U^{\alpha}$, together with property (4) from Section 4.1, would imply the existence of a proper subgroup $\tilde{U} \subset P^{\alpha}$ such that $\mu_{x}^{P^{\alpha}}$ is supported on $\tilde{U}$ a.e. However, by choice of $P^{\alpha}$, $P^{\alpha}$ is the smallest such subgroup. Hence $\mu$ is faithfully $P^{\alpha}$-recurrent.

Let $a_{\alpha}$ be as in Lemma 3.1. Then $a=a_{\alpha}^{-1}$ satisfies the assumption in Theorem 6.1. Therefore, Theorem 6.1 applies and we obtain one of two possible outcomes.

Assume (1) in Theorem 6.1 holds. Recall that by assumption on $\alpha$ (i.e. that it is a low entropy root), by (4.5) applied to $G^{-}$, and by assumption on $P^{\alpha}$ (i.e. that it supports $\mu_{x}^{P^{\alpha}}$ ) we have that the measure $\mu_{x}^{G^{-}}$is also supported on $P^{\alpha}$ a.e. Therefore, (3) from Section 4.1 shows that there exists some set $X^{\prime} \subset X$ of full measure so that $x, h . x \in X^{\prime}$ with $h \in G^{-}$implies $h \in P^{\alpha}$. By assumption $\mu$ is not $\left(\mu_{x}^{P^{\alpha}}, C_{G}\left(P^{\alpha}\right) \cap\right.$ $\left.G^{-}\right)$-transient, which together implies now that for every set of full measure $X^{\prime \prime} \subset X$ there exists some $x \in X^{\prime \prime}$ and $u \in C_{P^{\alpha}}\left(P^{\alpha}\right)$ so that both $x, u . x \in X^{\prime \prime}$ and $\mu_{x}^{P^{\alpha}}=\mu_{u . x}^{P^{\alpha}}$. However, by (3) from Section 4.1 again, this shows that for any set of full measure there exists some $x$ in that set and some nontrivial $u \in C_{P^{\alpha}}\left(P^{\alpha}\right)$ with $\mu_{x}^{P^{\alpha}} \propto \mu_{x}^{P^{\alpha}} u=u \mu_{x}^{P^{\alpha}}$. This actually implies, almost surely, that in fact $\mu_{x}^{P^{\alpha}}=\mu_{x}^{P^{\alpha}} u$. There are two ways to show this: if $\mu_{x}^{P^{\alpha}}=c \mu_{x}^{P^{\alpha}} u$ for $c>1$ then for every 
$n, \mu_{a} x^{P^{\alpha}}=c \mu_{a} \cdot x^{P^{\alpha}} a u a^{-1}$ which can be shown to contradict Poincaré recurrence for $a$ as $a^{n} u a^{-n} \rightarrow e$ (see [4, Lemma 5.10] for details). Alternatively, $\mu_{x}^{P^{\alpha}}=c \mu_{x}^{P^{\alpha}} u$ for $c>1$ implies that the $\mu_{x}^{P^{\alpha}}$-measure of a ball of radius $t$ around $e \in P^{\alpha}$ grows exponentially in $t$ which contradicts [9, Thm. 6.29].

Moreover, having found some nontrivial $u \in P^{\alpha}$ preserving $\mu_{x}^{P^{\alpha}}$, we claim that, almost surely, $\mu_{x}^{P^{\alpha}}$ is invariant under a nontrivial connected $A$-normalized subgroup $H_{x} \subset C_{P^{\alpha}}\left(P^{\alpha}\right)$. In fact this follows from [4, Prop. 6.2] (which once more only relies on Poincaré recurrence) together with the fact that the eigenvalues of the adjoint action of elements of $A$ belong to $\mathbb{Q}_{\sigma}$. For the same reason, $H_{x}$ is normalized by $A$ so that $\mu_{a . x}^{P^{\alpha}}$ is also invariant under $H_{x}$. Using ergodicity of $A$, it follows that $\mu_{y}^{P^{\alpha}}$ is invariant under a nontrivial connected $A$-normalized subgroup $H \subset C_{P^{\alpha}}\left(P^{\alpha}\right)$. We have shown statement (1) of the corollary.

If (1) in Theorem 6.1 holds for the root $-\alpha$, then this gives as above invariance of $\mu$ under a nontrivial connected subgroup $H \subset P^{-\alpha}$ which implies (1) of the corollary once more. Therefore, we can now assume that case (2) holds in Theorem 6.1 both for $\alpha$ and for $-\alpha$. In other words $\mu$ is $\left(\mu_{x}^{P^{\alpha}}, C_{G}\left(P^{\alpha}\right)\right)$-exceptional and $\left(\mu_{x}^{P^{-\alpha}}, C_{G}\left(P^{-\alpha}\right)\right)$ exceptional. We claim that this implies property (2) of the corollary.

Let $\epsilon>0$. Now choose $O$ to be a sufficiently small neighborhood of the identity in $G$ so that the natural map $O \rightarrow O \cdot x$ is injective for $x$ belonging to a set of measure bigger than $1-\epsilon / 3$. Then there exists $\delta>0$ and a set $B \subset X$ of measure $>1-\epsilon / 3$ so that $\mu_{x}^{P^{\alpha}}=\mu_{y}^{P^{\alpha}}$ with $x, y \in B$ and $d(x, y)<\delta$ implies $y \in\left(O \cap C_{G}\left(P^{\alpha}\right)\right)$. $x$. Replacing $B$ by an appropriately chosen subset of measure $>1-\epsilon$, we may assume that the same holds similarly for $\mu_{x}^{P^{-\alpha}}$ and $C_{G}\left(P^{-\alpha}\right)$, and moreover that the map $O \rightarrow O . x$ is injective for all $x \in B$.

Assume now $z$ is such that $\mu_{z}^{\mathcal{E}}(B)>0$, and $x \in B \cap \operatorname{supp}\left(\left.\mu_{z}^{\mathcal{E}}\right|_{B}\right)$.

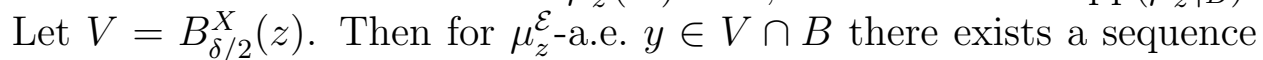
$a_{n}^{\prime} \in A^{\prime}$ with $a_{n}^{\prime} . y \in V \cap B$ and $a_{n}^{\prime} . y \rightarrow x$ as $n \rightarrow \infty$. By property (5) of Section 4.1 this implies almost surely that $\mu_{y}^{P^{\alpha}}=\mu_{a_{n}^{\prime} \cdot y}^{P^{\alpha}}$ and $\mu_{y}^{P^{-\alpha}}=\mu_{a_{n}^{\prime} . y}^{P^{-\alpha}}$. However, this implies that $a_{n}^{\prime} . y=c_{n} . y$ for some $c_{n} \in$ $O \cap C_{G}\left(P^{\alpha}\right) \cap C_{G}\left(P^{-\alpha}\right)$. Let $L=C_{G}\left(P^{\alpha}\right) \cap C_{G}\left(P^{-\alpha}\right)$. As $a_{n}^{\prime} \cdot y \rightarrow x$ as $n \rightarrow \infty$ and since we may assume that $O$ is a compact neighborhood of the identity, we may choose a subsequence so that $c_{n} \rightarrow c \in L$. Therefore, $\mu_{z_{0}}^{\mathcal{E}}$-a.e. $y \in V \cap B$ belong to L.x. As $P^{\alpha} \subset U^{\alpha}$ we have $A^{\prime} \subset C_{G}\left(P^{\alpha}\right)$ and similarly $A^{\prime} \subset C_{G}\left(P^{-\alpha}\right)$ and so $A^{\prime} \subset L$. This shows that the orbit L.x is an $A^{\prime}$-invariant set of positive $\mu_{z}^{\mathcal{E}}$-measure. Hence $\mu_{z}^{\mathcal{E}}$ is concentrated on the orbit L.x. Letting $\epsilon$ go to zero, we obtain 
that $\mu$-a.e. ergodic component w.r.t. $A^{\prime}$ is concentrated on a single $L$-orbit.

Finally, note that by Proposition 3.2 the Lie algebra of $C_{G}\left(P^{\alpha}\right)$ is contained in $\mathfrak{g}^{0} \oplus \sum_{(\beta, \alpha) \geq 0} \mathfrak{u}^{\beta}$ so the Lie algebra of $L=C_{G}\left(P^{\alpha}\right) \cap$ $C_{G}\left(P^{-\alpha}\right)$ is contained in $\mathfrak{g}^{0} \oplus \sum_{(\beta, \alpha)=0} \mathfrak{u}^{\beta}$ hence is centralized by $a_{\alpha}$.

\section{THE EXCEPTIONAL CASE AND RANK-ONE FACTORS}

In this section we will prove the following step towards Theorem 1.3.

Proposition 7.1. In the setting of Theorem 1.3 we have that (2) of Corollary 6.2 cannot hold.

We will need the following lemmas.

Lemma 7.2. Let $\mathbf{G}$ be a $\mathbb{Q}_{\sigma}$-almost simple algebraic group. Let $A$ be a maximal $\mathbb{Q}_{\sigma}$-split subtorus of $\mathbf{G}$ and let $\alpha \in \Phi$ be a Lyapunov root. Let $\mathbf{L}<\mathbf{G}$ be an algebraic subgroup that is A-normalized and contains both a one-parameter subgroup of $U^{\alpha}$ and a one-parameter subgroup of $U^{-\alpha}$. Then $\mathbf{L}$ also contains a power of the element $a_{\alpha}$ as in Lemma 3.1.

Proof. As $\mathbf{L}$ is $A$-normalized we may assume that the one-parameter subgroups are also $A$-normalized, i.e. that they are uniquely defined by some element $v^{+}$of $\mathfrak{u}^{\beta}$ with $\beta \in\{1,2\} \alpha$, resp. $v^{-}$of $\mathfrak{u}^{-\gamma}$ with $\gamma \in\{1,2\} \alpha$. Let $\mathfrak{l}$ be the Lie algebra of $\mathbf{L}$ and let $\mathbf{H}_{\alpha}$ be the algebraic subgroup generated by $U^{\alpha}$ and $U^{-\alpha}$.

We may now apply Proposition 3.2 to see that the Lie algebra of $\mathbf{L} \cap \mathbf{H}_{\alpha}$ is symmetric in dimensions, i.e. the Lie algebra intersected with $\mathfrak{u}^{\alpha}$ has the same dimension as the intersection with $\mathfrak{u}^{-\alpha}$ and similarly for $2 \alpha$. Also by Proposition 3.2, ad $v_{v^{+}}^{2}$ restricted to $\mathfrak{u}^{-\beta}$ is injective. However, this implies that there exists $w \in \mathfrak{l} \cap \mathfrak{u}^{-\alpha}$ with $\operatorname{ad}_{v^{+}}^{2}(w)=v_{+}$. Hence we can choose the $\mathfrak{s l}_{2}$-triple as in the proof of Lemma 3.1 and Proposition 3.2 within $\mathfrak{l}$. However, since $\mathbf{H}_{\alpha}$ is semi-simple with $\mathbb{Q}_{\sigma^{-}}$ rank one (which follows since $A$ is maximal), we see that the split element $\left[v_{+}, w\right] \in \mathfrak{g}^{0}$ must be in the Lie algebra of the torus generated by powers of $a_{\alpha}$. This implies that a power of $a_{\alpha}$ belongs to $\mathbf{L}$ as claimed.

Lemma 7.3. Let $\mathbb{G}, S, F^{\prime} \subset F, A, \Gamma, \mu$ be as in Theorem 1.1. Suppose $\mu$ is concentrated on the orbit $\Gamma L p$ where $L=\mathbb{L}\left(\mathbb{Q}_{S}\right)$ for some $\mathbb{Q}$ group $\mathbb{L}$ and $p \in \operatorname{supp} \mu$. Then there is a reductive $\mathbb{Q}$-groups $\mathbb{L}^{\prime}$ with $\mathbb{Q}$-anisotropic center so that $\mu$ is supported on the orbit $\Gamma L^{\prime} p$, where $L^{\prime}=\mathbb{L}^{\prime}\left(\mathbb{Q}_{S}\right)$.

Note that we do not assume $\Gamma L p$ is closed; it follows however from $\mathbb{L}^{\prime}$ having no $\mathbb{Q}$-characters that $\Gamma L^{\prime} p$ will be closed of finite volume. 
Proof. Conjugating $A$ if necessary and replacing $\mu$ by $\mu p^{-1}$ we may as well assume that $p=e$. Applying Lemma 2.1, by passing to a normal subgroup of $\mathbb{L}$ if necessary, we may assume that $\mathbb{L}$ has no $\mathbb{Q}$ characters, so that $\Gamma L$ is closed of finite volume, and supports $\mu$. It remains, however, to show that $\mathbb{L}$ can be taken to be reductive.

Suppose $\mathbb{L}$ has a unipotent radical $\mathbb{U}$, which is automatically defined over $\mathbb{Q}$. As $A<\mathbb{L}\left(\mathbb{Q}_{S}\right)$, we know that $A$ normalizes $\mathbb{U}\left(\mathbb{Q}_{S}\right)$ and its (generalized) Lie algebra. For a given $\sigma \in S$ this implies that $\mathbb{U}\left(\mathbb{Q}_{\sigma}\right)$ must be a direct product of the subgroups $\mathbb{U}\left(\mathbb{Q}_{\sigma}\right) \cap G_{f}$ with $f \in F^{\prime} \cap F_{\sigma}$ and a subgroup $U^{\prime} \subset \prod_{f \in F_{\sigma} \backslash F^{\prime}} G_{f}$. Indeed the Lie algebra $\mathfrak{f}$ of $\mathbb{U}$ (considered over $\mathbb{Q}_{\sigma}$ ) is $A_{f}$-normalized for all $f \in F^{\prime} \cap F_{\sigma}$. Therefore,

$$
\mathfrak{f}=\sum_{\alpha} \mathfrak{f} \cap \mathfrak{u}^{\alpha}+\mathfrak{f} \cap \mathfrak{g}^{0} .
$$

However, $\mathfrak{f} \cap \mathfrak{g}^{0}$ projected to $\sum_{f \in F^{\prime}} \mathfrak{g}_{f}^{0}$ must be trivial as the latter cannot contain the Lie algebra of a unipotent subgroup. Hence $\mathfrak{f} \cap \mathfrak{g}^{0}$ belongs to $\sum_{f \in F_{\sigma} \backslash F^{\prime}} \mathfrak{g}_{f}$.

We claim that $\mathfrak{f} \cap \mathfrak{u}^{\alpha}$ is trivial for all $\alpha \in \Phi^{\prime}$. Suppose this were false. Then the Lie algebra $\mathfrak{h}=\mathfrak{f} \cap \mathfrak{g}_{f}$ is $A_{f}$-normalized and the determinant of the adjoint representation of $A_{f}$ on $\mathfrak{f}$ must be one (as this determinant is a $\mathbb{Q}$-character of $\mathbb{L}$ which is assumed to be trivial). However, this enables us to use the same arguments as in the proof of Theorem 5.1 (which relies on Proposition 3.2) to show that the dimensions of the intersections $\mathfrak{f} \cap \mathfrak{u}^{\alpha}$ are symmetric. In particular non-triviality of $\mathfrak{f} \cap \mathfrak{u}^{\alpha}$ for some root would force non-triviality of $\mathfrak{f} \cap \mathfrak{u}^{-\alpha}$ and also of $\mathfrak{f} \cap \mathfrak{g}^{0} \cap \mathfrak{g}_{f}$. However, we already argued that the latter must be trivial.

Therefore $\mathbb{U}\left(\mathbb{Q}_{\sigma}\right) \cap G_{f}$ must be trivial for $f \in F^{\prime} \cap F_{\sigma}$ and all $\sigma \in S$, hence $\mathbb{U}\left(\mathbb{Q}_{\sigma}\right)$ commutes with $A$. Let now $\mathbb{L}^{\prime}<\mathbb{L}$ be a $\mathbb{Q}$-Levy subgroup of $\mathbb{L}$. Since $\mathbb{L}^{\prime}$ is unique up to conjugation by $\mathbb{U}$ and $A$ commutes with $\mathbb{U}$ we see that any choice gives that $\mathbb{L}^{\prime}$ contains $A$. Since $\Gamma L^{\prime}$ is closed and $A$-invariant, and since we assumed that $e \in \operatorname{supp} \mu$, it follows from ergodicity of $A$ that $\mu$ is supported on $\Gamma L^{\prime}$.

We say that an element $a \in A$ is of class $\mathcal{A}$ if $a \in G_{\sigma}$ for some $\sigma \in S$ and all eigenvalues of $a_{\sigma}$ are powers of some $t \in \mathbb{Q}_{\sigma}$ with $|t| \neq 1$. The importance of this condition is that if $a$ is of class $\mathcal{A}$ then for any algebraic representation $\rho$ of $G_{\sigma}$ on any $\mathbb{Q}_{\sigma}$-vector space $V$ and any vector $v \in V$, the sequence $\rho\left(a^{n}\right) v$ converges in the projective sense to an eigendirection of $\rho(a)$.

Proof of Proposition 7.1. Let $H=C_{G}\left(P^{\alpha}\right) \cap C_{G}\left(P^{-\alpha}\right) \subset C_{G}\left(a_{\alpha}\right)$ be as in case (2) of Corollary 6.2. Let $x=\Gamma g$ be a point for which the conclusion of the corollary holds. We define $\Lambda_{g}=\Gamma \cap\left(g \mathrm{Hg}^{-1}\right)$, 
take $\mathbb{M}_{g}$ to be the the connected component of its Zariski closure, i.e. $\mathbb{M}_{g}=\left(\bar{\Lambda}_{g}\right)^{\circ}$, and finally define

$$
\mathbb{L}_{g}=\left[C_{\mathbb{G}}\left(\mathbb{M}_{g}\right)^{\circ}, C_{\mathbb{G}}\left(\mathbb{M}_{g}\right)^{\circ}\right] .
$$

By definition we have that $g P^{\alpha} g^{-1}, g P^{-\alpha} g^{-1}$ are subgroups of the $\mathbb{Q}_{\sigma}$-points of $C_{\mathbb{G}}\left(\mathbb{M}_{g}\right)^{\circ}$ with $\sigma \in S$ the place corresponding to the root $\alpha$, which implies $g P^{\alpha} g^{-1}, g P^{-\alpha} g^{-1}<\mathbb{L}_{g}\left(\mathbb{Q}_{\sigma}\right)$. By Lemma 7.2 this implies that a power of $g a_{\alpha} g^{-1}$ also belongs to $\mathbb{L}_{g}\left(\mathbb{Q}_{\sigma}\right)$.

Let us study how these $\mathbb{Q}$-groups vary: Since $A$ normalizes $P^{\alpha}$ and $P^{-\alpha}$ it follows that $A$ also normalizes $H$. Therefore, $g H g^{-1}=$ ( $g a) H(g a)^{-1}$ for any $a \in A$. From this we get that $\Lambda_{g}=\Lambda_{g a}$ for any $g \in G$ and hence the same holds for the groups $\mathbb{M}_{g}$ and $\mathbb{L}_{g}$ that are determined by $\Lambda_{g}$.

Choose in some measurable way a representative $g_{x} \in G$ for every $x \in X$ so that $x=\Gamma g_{x}$. Since $\mathbb{G}$ has only countably many $\mathbb{Q}$-subgroups, there is a subset $Z \subset X$ of positive $\mu$-measure on which $\mathbb{M}_{g_{x}}, \mathbb{L}_{g_{x}}$ are constant, say $\mathbb{L}_{g_{x}}=\mathbb{L}$ and $\mathbb{M}_{g_{x}}=\mathbb{M}$. By ergodicity, for $\mu$ a.e. $x \in X$ there is some $a \in A$ for which $x a \in Z$, hence we see that in fact almost every point $x$ can be written as $x=\Gamma g$ for some $g$ for which $\mathbb{M}=\mathbb{M}_{g}$ and $\mathbb{L}=\mathbb{L}_{g}$.

Set $A^{\prime}=\operatorname{ker} \alpha<A$. We claim that $g A^{\prime} g^{-1}<\mathbb{M}$ almost surely. Indeed, assume that the $A^{\prime}$-ergodic component of $\mu$ at $x=\Gamma g$ is concentrated on the single $H$-orbit $x H=\Gamma g H$ as in case (2) in Corollary 6.2 - currently we don't know whether this orbit is closed. Let $a \in A^{\prime} \cap G_{\sigma}$ be an element of class $\mathcal{A}$, and let $x \in \operatorname{supp} \mu$ satisfy Poincaré recurrence applied to $a$ and neighborhoods of $x$ within the $H$-orbit (this condition holds for $\mu$-a.e. $x \in X$ by the above assumption on $A^{\prime}$-ergodic components). Then there exists a sequence $\gamma_{k} g a^{n_{k}}=g h_{k}$ as $k \rightarrow \infty$ with $\gamma_{k} \in \Gamma, h_{k} \in H$, and $h_{k} \rightarrow e$ as $k \rightarrow \infty$. As $a \in A^{\prime}<H$ we get $\gamma_{k} \in \Lambda_{g}=\Gamma \cap g H g^{-1}$. By Chevalley's theorem there exists an algebraic representation $\rho$ of $\mathbb{G}$ over $\mathbb{Q}_{\sigma}$ and a vector $v$ that together define the Zariski closure of $\Lambda_{g}$ as the stabilizer of the line generated by $v$. Notice that $\rho\left(g a^{-n_{k}} g^{-1}\right) v$ converges projectively to an eigenvector since $a$ is of class $\mathcal{A}$. As $h_{k}$ converges to the identity, we also get that $\rho\left(g h_{k} a^{-n_{k}} g^{-1}\right) v=\rho\left(\gamma_{k}\right) v$ converges projectively to an eigenvector of $\rho(a)$. However, as $\rho\left(\gamma_{k}\right) v \in \mathbb{Q}_{\sigma} v$ for all $k$ this shows that $v$ is an eigenvector of $\rho(a)$ and that $a$ belongs to the Zariski closure of $\Lambda_{g}$ as claimed. As this holds for all $a \in A^{\prime}$ that are of class $\mathcal{A}$, we get $g A^{\prime} g^{-1}<\mathbb{M}\left(\mathbb{Q}_{S}\right)$.

Since the group $\mathbb{L}$ is defined over $\mathbb{Q}$, so is its unit normalizer $\mathbb{N}=$ $N_{\mathbb{G}}^{1}(\mathbb{L})$. In fact, by its very definition, $\mathbb{N}$ fixes a vector in a $\mathbb{Q}$-representation of $\mathbb{G}$ (namely the vector corresponding to the Lie algebra of 
$\mathbb{L}$ in an appropriate wedge product of the Lie algebra of $\mathbb{G}$ ). Since $\Gamma$ is commensurable with $\mathbb{G}\left(\mathcal{O}_{S}\right)$, it follows that $\Gamma \mathbb{N}\left(\mathbb{Q}_{S}\right)$ is closed in $X$. Moreover we have seen that $g A^{\prime} g^{-1}$ commutes with $\mathbb{L}\left(\mathbb{Q}_{S}\right)$ (since it is contained in $\mathbb{M})$ hence $g A^{\prime} g^{-1}<\mathbb{N}\left(\mathbb{Q}_{S}\right)$. We have already noted that $g a_{\alpha}^{\ell} g \in \mathbb{N}\left(\mathbb{Q}_{S}\right)$ for some $\ell$. Hence a finite index subgroup of $g \mathrm{Ag}^{-1}$ is contained in $\mathbb{N}\left(\mathbb{Q}_{S}\right)$, and since $A$ is a product of points of connected algebraic groups we actually get that $g A g^{-1}<\mathbb{N}\left(\mathbb{Q}_{S}\right)$.

Since $\mu$ is $A$-ergodic, and $\Gamma g \in \operatorname{supp} \mu$, we may conclude that $\mu$ is supported on $\Gamma \mathbb{N}\left(\mathbb{Q}_{S}\right) g$. By Lemma 7.3 and the minimality assumption on $\mathbb{G}$ in Theorem 1.3, we may conclude that $\mathbb{N}=\mathbb{G}$, but since $\mathbb{L}$ is a nontrivial normal subgroup of $\mathbb{N}$ this contradicts the assumption in Theorem 1.3 that $\mathbb{G}$ is almost simple.

\section{UNIPOTENT INVARIANCE}

In this section we will finish the proof of Theorem 1.3. By Proposition 7.1 we know that case (2) of Corollary 6.2 never takes place. Knowing that $\mu$ is invariant under a unipotent subgroup we are going to use the classification of measures that are invariant under unipotent one-parameter subgroups as proven by Ratner [30, 31] and extended by Ratner [33] and Margulis and Tomanov [24]. In fact, it will be convenient to use a refined version of these results by Tomanov [37] that is more adapted to the arithmetic case (i.e., when the lattice $\Gamma$ is commensurable to $\mathbb{G}\left(\mathbb{Q}_{S}\right)$ ).

Proof of Theorem 1.3. In the case of a high entropy root $\alpha$ we have seen in Theorem 5.1 that $\mu$ is invariant under a nontrivial subgroup of $U^{\alpha}$. In the case of a low entropy root $\alpha$ by Proposition 7.1 we know that the first possibility (invariance) in Corollary 6.2 holds, which says that $\mu$ is invariant either under a nontrivial subgroup of $U^{\alpha}$ or of $U^{-\alpha}$.

Let now $H_{u}$ be the subgroup of $G$ generated by all connected subgroups of $U^{\alpha}$ for any $\alpha \in \Phi^{\prime}$ that preserve $\mu$. As $H_{u}$ is generated by unipotent one-parameter subgroups we wish to apply the $S$-algebraic version of Ratner's measure classification theorem. However, we do not know whether $\mu$ is ergodic under the $H_{u}$-action, so we have to apply the classification to the $H_{u}$-ergodic components $\mu_{x}^{\mathcal{E}}$ where $\mathcal{E}$ is the $\sigma$ algebra of $H_{u}$-invariant Borel subsets of $X$ and analyze how this affects the measure $\mu$ (a similar, more general, analysis can be found in [25]; however, for completeness we include the argument here).

Let $x=\Gamma g$ be such that the conditional measure $\mu_{x}^{\mathcal{E}}$ is an $H_{u^{-}}$ invariant and ergodic probability measure. By [33] or [24] $\mu_{x}^{\mathcal{E}}$ is the normalized $L_{x}$-invariant volume measure on a periodic orbit $x L_{x}$ of a closed subgroup $L_{x}<G$ and [37] gives us the additional information 
that there exists a connected $\mathbb{Q}$-group $\mathbb{L}_{g}$ so that $L_{x}$ is a finite index subgroup of $g^{-1} \mathbb{L}_{g}\left(\mathbb{Q}_{S}\right) g$ containing $H_{u}$. Note that the group $L_{x}$ may not be unique as e.g. a finite index subgroup would have the same property, but that its (generalized) Lie algebra $\mathfrak{l}_{x}$ is well defined.

Let us analyze the dependence of the above groups on the base points. Since $H_{u}$ is $A$-normalized we see that the $\sigma$-algebra $\mathcal{E}$ is invariant under $A$ and so the conditional measure $\mu_{x}^{\mathcal{E}}$ is mapped a.s. under $a$ to $\mu_{a . x}^{\mathcal{E}}$. This implies that $a L_{x} a^{-1}$ is commensurable with $L_{a . x}$ and that $\operatorname{Ad}_{a}\left(\mathfrak{l}_{x}\right)=\mathfrak{l}_{a . x}$. Now choose $a \in A$ to be of class $\mathcal{A}$ and apply Poincaré recurrence. It follows that along some subsequence $\mathfrak{l}_{a^{n_{k}} \cdot x}$ should converge to $\mathfrak{l}_{x}$, but since $a$ is of class $\mathcal{A}$ any limit point of $\mathfrak{l}_{a^{n} . x}=\operatorname{Ad}_{a^{n}}\left(\mathfrak{l}_{x}\right)$ has to be normalized by $a$. Since $A$ is generated by its elements of class $\mathcal{A}$, we see that $\mathfrak{l}_{x}$ is constant along the $A$-orbit and by ergodicity constant $\mu$-a.e. Let us denote the common value by $\mathfrak{l}_{0}$. This implies that every point $x \in X$ has a representative $g_{x} \in G$ so that $x=\Gamma g_{x}$ and such that the Lie algebra of $g_{x}^{-1} \mathbb{L}_{g_{x}}\left(\mathbb{Q}_{S}\right) g_{x}$ equals $\mathfrak{l}_{0}$; and it is moreover clear that we can make such a choice so that the map $x \mapsto g_{x}$ is measurable. Since there are only countably many $\mathbb{Q}$-subgroups of $\mathbb{G}$ there is some subset $Z \subset X$ of positive $\mu$ measure and a connected $\mathbb{Q}$-subgroup $\mathbb{L}<\mathbb{G}$ so that $\mathbb{L}_{g_{x}}=\mathbb{L}$ for every $x \in Z$. However by definition of $g_{x}$ the Lie algebra of $g_{x}^{-1} \mathbb{L}\left(\mathbb{Q}_{S}\right) g_{x}=\mathfrak{l}_{0}$ for every $x \in Z$. Set $\mathbb{M}=N_{\mathbb{G}}(\mathbb{L})$. Since the Lie algebra determines the group (for connected algebraic groups), it follows that $g_{x} \in \mathbb{M}\left(\mathbb{Q}_{S}\right) g_{x^{\prime}}$ for every $x, x^{\prime} \in Z$.

Since $\mathfrak{l}_{0}$ is normalized by $A$, we have that $g_{x} A g_{x}^{-1}<\mathbb{M}\left(\mathbb{Q}_{S}\right)$ for $x \in Z$ hence fixing some $x_{0} \in Z$ we conclude that $\Gamma \mathbb{M} g_{x_{0}}$ is an $A$-invariant set of positive $\mu$ measure (it contains $Z$ ), so by ergodicity $\mu$ is concentrated on this set.

Applying Lemma 7.3, and using the minimality condition on $\mathbb{G}$ in the statement of Theorem 1.3 , the only possibility that remains is $\mathbb{G}=$ $\mathbb{M}$. Since $\mathbb{G}$ is $\mathbb{Q}$-almost simple and $\mathbb{L}$ is a nontrivial $\mathbb{Q}$-group with $\mathbb{L} \triangleleft \mathbb{M}=\mathbb{G}$ we must have that $\mathbb{L}=\mathbb{G}$.

We conclude that each $H_{u}$-ergodic component $\mu_{x}^{\mathcal{E}}$ is invariant under a finite index subgroup $L_{x}$ of $\mathbb{G}\left(\mathbb{Q}_{S}\right)$. Set

$$
\tilde{L}=\bigcap_{a \in A /\left(A \cap L_{x}\right)} a L_{x} a^{-1} ;
$$

note that the intersection is finite since $A \cap L_{x}$ is of finite index in $A$. Then $\tilde{L}$ is also a finite index subgroup of $\mathbb{G}\left(\mathbb{Q}_{S}\right)$ and for every $a \in A$ we have that $\mu_{a . x}^{\mathcal{E}}$ is $\tilde{L}$-invariant, hence by ergodicity $\mu$ is $\tilde{L}$ invariant. Since $\mu$ is also $A$-invariant, $\mu$ is $L=\tilde{L} A$-invariant. By ergodicity, $\mu$ is supported on a single $L$-orbit. 


\section{REFERENCES}

[1] J. W. S. Cassels and H. P. F. Swinnerton-Dyer. On the product of three homogeneous linear forms and the indefinite ternary quadratic forms. Philos. Trans. Roy. Soc. London. Ser. A., 248:73-96, 1955.

[2] S. G. Dani and G. A. Margulis. Orbit closures of generic unipotent flows on homogeneous spaces of SL(3, R). Math. Ann., 286(1-3):101-128, 1990.

[3] M. Einsiedler, A. Katok, Invariant measures on $G / \Gamma$ for split simple Lie groups $G$. Dedicated to the memory of Jürgen K. Moser. Comm. Pure Appl. Math. 56 (2003), no. 8, 1184-1221.

[4] M. Einsiedler and A. Katok, Rigidity of measures - the high entropy case, and non-commuting foliations, Probability in mathematics. Israel J. Math. 148 (2005), 169-238.

[5] M. Einsiedler, A. Katok, E. Lindenstrauss, Invariant measures and the set of exceptions to Littlewood's conjecture, Ann. of Math. (2) 164 (2006), no. 2, $513-560$.

[6] M. Einsiedler, E. Lindenstrauss, Joinings of higher-rank diagonalizable actions on locally homogeneous spaces. Duke Math. J. 138 (2007), no. 2, 203-232.

[7] M. Einsiedler and E. Lindenstrauss. Diagonalizable flows on locally homogeneous spaces and number theory. In International Congress of Mathematicians. Vol. II, pages 1731-1759. Eur. Math. Soc., Zürich, 2006.

[8] M. Einsiedler, E. Lindenstrauss. On measures invariant under diagonalizable actions: the rank-one case and the general low-entropy method, J. Mod. Dyn. 2 (2008), no. 1, 83-128.

[9] M. Einsiedler, E. Lindenstrauss, Diagonal actions on locally homogeneous spaces. Homogeneous flows, moduli spaces and arithmetic, 155-241, Clay Math. Proc., 10, Amer. Math. Soc., Providence, RI, 2010.

[10] M. Einsiedler, E. Lindenstrauss, Ph. Michel, and A. Venkatesh, Distribution of periodic torus orbits on homogeneous spaces. Duke Math. J. 148 (2009), no. $1,119-174$.

[11] M. Einsiedler, E. Lindenstrauss, Ph. Michel, and A. Venkatesh. Distribution of periodic torus orbits and Duke's theorem for cubic fields. Ann. of Math. (2), 173(2):815-885, 2011.

[12] H. Furstenberg. Disjointness in ergodic theory, minimal sets, and a problem in Diophantine approximation. Math. Systems Theory, 1:1-49, 1967.

[13] N. Jacobson, Lie algebras, Interscience Tracts in Pure and Applied Mathematics, No. 10, Interscience Publishers (a division of John Wiley \& Sons), New York-London, 1962, ix +331 .

[14] A. Johnson. Measures on the circle invariant under multiplication by a nonlacunary subsemigroup of the integers. Israel J. Math., 77(1-2):211-240, 1992.

[15] A. Johnson and D. Rudolph. Convergence under $\times_{q}$ of $\times_{p}$ invariant measures on the circle. Adv. Math., 115(1):117-140, 1995.

[16] B. Kalinin and A. Katok, Invariant measures for actions of higher rank abelian groups, Smooth ergodic theory and its applications (Seattle, WA, 1999), Amer. Math. Soc., Providence, RI, 2001, pp. 593-637.

[17] A. Katok and R. Spatzier, Invariant measures for higher-rank hyperbolic abelian actions, Ergodic Theory Dynam. Systems 16 (1996), no. 4, 751-778. Corrections: Ergodic Theory Dynam. Systems 18(1998) no. 2, 503-507. 
[18] D. Kleinbock. Nondense orbits of flows on homogeneous spaces. Ergodic Theory Dynam. Systems, 18(2):373-396, 1998.

[19] A. Knapp, Lie groups beyond an introduction. Progress in Mathematics, 140. Birkhuser Boston, Inc., Boston, MA, 1996. xvi+604 pp.

[20] E. Lindenstrauss, Invariant measures and arithmetic quantum unique ergodicity, Ann. of Math. (2) 163 (2006), no. 1, 165-219.

[21] Russell Lyons. On measures simultaneously 2- and 3-invariant. Israel J. Math., 61(2):219-224, 1988.

[22] G.A. Margulis, Discrete subgroups of semisimple Lie groups, Ergebnisse der Mathematik und ihrer Grenzgebiete (3) [Results in Mathematics and Related Areas (3)], 17. Springer-Verlag, Berlin, 1991. $x+388$ pp.

[23] G. A. Margulis. Oppenheim conjecture. In Fields Medallists' lectures, volume 5 of World Sci. Ser. 20th Century Math., pages 272-327. World Sci. Publishing, River Edge, NJ, 1997.

[24] G. A. Margulis and G. M. Tomanov, Invariant measures for actions of unipotent groups over local fields on homogeneous spaces, Invent. Math. 116 (1994), no. 1-3, 347-392.

[25] G.A. Margulis, G.M. Tomanov, Measure rigidity for almost linear groups and its applications. J. Anal. Math. 69 (1996), 25-54.

[26] D. Prasad, An analogue of a conjecture of Mazur: a question in Diophantine approximation on tori. Contributions to automorphic forms, geometry, and number theory, 699-709, Johns Hopkins Univ. Press, Baltimore, MD, 2004.

[27] M. Ratner. Factors of horocycle flows. Ergodic Theory Dynam. Systems, 2(34):465-489, 1982.

[28] M. Ratner. Rigidity of horocycle flows. Ann. of Math. (2), 115(3):597-614, 1982.

[29] M. Ratner. Horocycle flows, joinings and rigidity of products. Ann. of Math. (2), 118(2):277-313, 1983.

[30] M. Ratner. On measure rigidity of unipotent subgroups of semisimple groups. Acta Math., 165(3-4):229-309, 1990.

[31] M. Ratner. On Raghunathan's measure conjecture. Ann. of Math. (2), 134(3):545-607, 1991.

[32] M. Ratner. Raghunathan's topological conjecture and distributions of unipotent flows. Duke Math. J., 63(1):235-280, 1991.

[33] M. Ratner, Raghunathan's conjectures for Cartesian products of real and padic Lie groups. Duke Math. J. 77 (1995), no. 2, 275-382.

[34] M. Rees. Some $R^{2}$-anosov flows. unpublished, 1982.

[35] D. Rudolph. $\times 2$ and $\times 3$ invariant measures and entropy. Ergodic Theory Dynam. Systems, 10(2):395-406, 1990.

[36] T. A. Springer, Linear algebraic groups, Second edition, Birkhäuser Boston, Boston, MA, 1998

[37] G. Tomanov, Orbits on homogeneous spaces of arithmetic origin and approximations. Analysis on homogeneous spaces and representation theory of Lie groups, OkayamaKyoto (1997), 265-297, Adv. Stud. Pure Math., 26, Math. Soc. Japan, Tokyo, 2000.

[38] G. Tomanov, Actions of maximal tori on homogeneous spaces. Rigidity in dynamics and geometry (Cambridge, 2000), 407-424, Springer, Berlin, 2002. 
[39] V. S. Varadarajan, Groups of automorphisms of Borel spaces, Trans. Amer. Math. Soc. 109 (1963), 191-220.

[40] M. Waldschmidt, Open Diophantine problems. Mosc. Math. J. 4(2004), no. 1, pp. 245-305, 312 .

(M. E.) ETH ZÜRich, RÄMistrasse 101 CH-8092 ZÜRICH Switzerland

E-mail address: manfred.einsiedler@math.ethz.ch

(E. L.) The Einstein Institute of Mathematics, Edmond J. Safra Campus, Givat Ram, The Hebrew University of Jerusalem Jerusalem, 91904, ISRAEL

E-mail address: elon@math.huji.ac.il 